\title{
Finite-Time Synchronization for Complex-Valued Recurrent Neural Networks with Time Delays
}

\author{
Ziye Zhang ${ }^{(D)},{ }^{1,2}$ Xiaoping Liu, ${ }^{3}$ Chong Lin ${ }^{(D)}{ }^{2}$ and Bing Chen ${ }^{2}$ \\ ${ }^{1}$ College of Mathematics and Systems Science, Shandong University of Science and Technology, Qingdao 266590, China \\ ${ }^{2}$ Institute of Complexity Science, Qingdao University, Qingdao 266071, China \\ ${ }^{3}$ Department of Electrical Engineering, Lakehead University, Thunder Bay, Canada ON P7B 5E1 \\ Correspondence should be addressed to Ziye Zhang; zhangzy02@126.com
}

Received 24 April 2018; Accepted 1 November 2018; Published 2 December 2018

Academic Editor: Dan Selişteanu

Copyright (C) 2018 Ziye Zhang et al. This is an open access article distributed under the Creative Commons Attribution License, which permits unrestricted use, distribution, and reproduction in any medium, provided the original work is properly cited.

\begin{abstract}
This paper focuses on the finite-time synchronization analysis for complex-valued recurrent neural networks with time delays. First, two kinds of common activation functions appearing in the existing references are combined together and more general assumptions are given. To achieve our aim, a nonlinear delayed controller with two independent parameters different from the existing ones is provided, which leads to great difficulty. To overcome it, a newly developed inequality is used. Then, via Lyapunov function approach, some criteria are derived to guarantee the finite-time synchronization of the considered system, and the settling time for synchronization is also estimated. Finally, two numerical simulations are given to support the effectiveness and advantages of the obtained results.
\end{abstract}

\section{Introduction}

In recent years, neural networks have been always very important to researchers in various fields, to name a few, control theory and signal processing in electrical engineering, parallel computation and pattern recognition in computer science, and modeling optimization problems in applied mathematics. Moreover, as everyone knows, most applications rely heavily on the dynamical behavior of recurrent neural networks. This sets off a research upsurge for the dynamics of neural networks. As a result, many researchers have put their efforts into the analysis and synthesis problems for the dynamics of neural networks for several decades; see [1-8] and the references therein.

In most results considering the stability and the stabilization of neural networks, the convergent mode is asymptotically stable and the time for system trajectories to reach an equilibrium point is infinite. However, in practical problems, the convergent time is often required to be faster or even finite. Thus, so as to satisfy this requirement, the topic about finite-time stability appeared. Recently, finite-time stability and synthesis problems for various systems have been hot issues and attracted many scholars' interests [9-29]. Among them, finite-time stability, stabilization, synchronization and robust passive control for time-delay systems, stochastic systems, chaotic systems, multiagent systems, and nonlinear systems were studied in [10-20]. For various neural networks models, finite-time stabilization, instabilizability, adaptive control, $H_{\infty}$ control, and state estimation were discussed in [22-26] and finite-time synchronization control problems were addressed in [27-29].

On the other hand, a complex-valued recurrent neural networks model has produced a research upsurge for the past few years. The main feature is that it owns complexvalued states, connection weights, and activation functions. This decides that this kind of system can be applied to a wider range involving electromagnetic waves, radar imaging, quantum waves, and so on, but it also leads to more difficulties in analyzing its dynamical behaviors in contrast to real-valued systems. In a word, anyway, the occurrence of complexvalued neural networks draws a great deal of attention from all aspects. Accordingly, many fruitful achievements have been reported for this model [30-52]. For example, global stability, global exponential stability, and Hopf bifurcation problems for complex-valued neural networks systems with delays or without delays were investigated in [32-40]. Dissipativity, 
passivity, state estimation, exponential stability, and inputto-state stability for memristor-based complex-valued neural networks with or without delays were discussed and relative criteria were established in [43-48]. However, for finite-time stability analysis of delayed complex-valued neural networks models, until now, few results have been developed. The boundedness problem of these systems in finite-time interval is considered in $[49,50]$. The finite-time synchronization problem for complex-valued neural networks (CVNNs) with infinite-time distributed delays is discussed in [51] and the finite-time stabilizability and instabilizability for complexvalued memristive neural networks with time delays are studied in [52]. Moreover, considering the rich background of complex-valued neural networks models and the present status of development for them, it is absolutely essential to explore the dynamics of these models deeply. Thus, these further inspire us to make deeper research.

In this article, in view of the unavoidability of time delays [53-55], the finite-time synchronization problem for complex-valued recurrent neural networks with time delays is presented. The main contributions of our work are embodied in several points: (1) A nonlinear delayed controller with independent parameters $\mu_{1}$ and $\mu_{2}$ different from the most existing results is designed to achieve the finite-time synchronization of the considered system. (2) To deal with the difficulty involved by the contribution (1), a new inequality stated in Lemma 6 proposed and proved by us in [52] is used. (3) For real-imaginary separate-type activation functions, two kinds of common functions in the existing references are combined together and more general conditions are assumed in this paper. So the proposed results have broader applications.

The rest of this article is organized as follows. Section 2 provides some preliminaries. Section 3 presents main criteria about the finite-time synchronization for delayed complexvalued recurrent neural networks and estimates the settling time by a new controller. Section 4 gives two numerical simulations to show the validity of theoretical results. Section 5 concludes this paper.

Notation. Throughout this paper, $i=\sqrt{-1}$ denotes the imaginary unit. For a vector $\xi=\left[\xi_{1}, \xi_{2}, \ldots, \xi_{n}\right]^{T} \in R^{n}, \xi$ is named a positive vector if $\xi_{l}>0, l=1,2, \ldots, n$.

\section{Preliminaries}

Consider a complex-valued recurrent neural networks model as the drive system represented by

$$
\begin{aligned}
\dot{z}_{p}(t)= & -d_{p} z_{p}(t)+\sum_{q=1}^{n} a_{p q} h_{q}\left(z_{q}(t)\right) \\
& +\sum_{q=1}^{n} b_{p q} m_{q}\left(z_{q}\left(t-\tau_{q}\right)\right)+J_{p}(t)
\end{aligned}
$$

with the initial conditions

$$
z_{p}(\theta)=\psi_{p}(\theta), \quad \theta \in[-\tau, 0]
$$

where $p=1,2, \ldots, n, n$ denotes the number of neurons; $z_{p}(t)$ is the state variable; $d_{p}>0$ is a constant; $a_{p q}$ and $b_{p q}$ are complex-valued connection weights; $J_{p}(t)$ represents external input vector; $\tau_{q}$ is the time delay; $\tau=\max \left\{\tau_{1}, \tau_{2}, \ldots, \tau_{n}\right\}$; $\psi_{p}(\theta) \in C([-\tau, 0], \mathbb{C}) ; h_{q}\left(z_{q}(t)\right)$ and $m_{q}\left(z_{q}\left(t-\tau_{q}\right)\right)$ are complex-valued activation functions.

Let $h_{q}\left(z_{q}(t)\right)=h_{q}^{R}\left(x_{q}(t), y_{q}(t)\right)+i h_{q}^{I}\left(x_{q}(t), y_{q}(t)\right)$ and $m_{q}\left(z_{q}\left(t-\tau_{q}\right)\right)=m_{q}^{R}\left(x_{q}\left(t-\tau_{q}\right), y_{q}\left(t-\tau_{q}\right)\right)+i m_{q}^{I}\left(x_{q}(t-\right.$ $\left.\left.\tau_{q}\right), y_{q}\left(t-\tau_{q}\right)\right)$. For simplicity, set $x_{q}=x_{q}(t), y_{q}=y_{q}(t)$, $x_{q}^{\tau_{q}}=x_{q}\left(t-\tau_{q}\right), y_{q}^{\tau_{q}}=y_{q}\left(t-\tau_{q}\right), \tilde{x}_{q}=\tilde{x}_{q}(t), \tilde{y}_{q}=\tilde{y}_{q}(t)$, $\tilde{x}_{q}^{\tau_{q}}=\tilde{x}_{q}\left(t-\tau_{q}\right)$, and $\tilde{y}_{q}^{\tau_{q}}=\tilde{y}_{q}\left(t-\tau_{q}\right)$. The complex-valued activation functions $h_{q}\left(z_{q}(t)\right)$ and $m_{q}\left(z_{q}\left(t-\tau_{q}\right)\right)$ are assumed to satisfy the following assumption.

Assumption 1. For any $x_{q}, \tilde{x}_{q}, y_{q}, \tilde{y}_{q}$ and $x_{q}^{\tau_{q}}, \tilde{x}_{q}^{\tau_{q}}, y_{q}^{\tau_{q}}, \tilde{y}_{q}^{\tau_{q}}$, there exist scalars $\kappa_{q}^{R R}, \kappa_{q}^{R I}, \kappa_{q}^{I R}, \kappa_{q}^{I I} \geq 0$ and $\pi_{q}^{R R}, \pi_{q}^{R I}, \pi_{q}^{I R}, \pi_{q}^{I I} \geq 0$ such that the following inequalities hold:

$$
\begin{aligned}
& \left|h_{q}^{R}\left(\tilde{x}_{q}, \tilde{y}_{q}\right)-h_{q}^{R}\left(x_{q}, y_{q}\right)\right| \\
& \quad \leq \kappa_{q}^{R R}\left|\tilde{x}_{q}-x_{q}\right|+\kappa_{q}^{R I}\left|\tilde{y}_{q}-y_{q}\right| \\
& \left|h_{q}^{I}\left(\tilde{x}_{q}, \tilde{y}_{q}\right)-h_{q}^{I}\left(x_{q}, y_{q}\right)\right| \\
& \quad \leq \kappa_{q}^{I R}\left|\tilde{x}_{q}-x_{q}\right|+\kappa_{q}^{I I}\left|\tilde{y}_{q}-y_{q}\right| \\
& \left|m_{q}^{R}\left(\tilde{x}_{q}^{\tau_{q}}, \tilde{y}_{q}^{\tau_{q}}\right)-m_{q}^{R}\left(x_{q}^{\tau_{q}}, y_{q}^{\tau_{q}}\right)\right| \\
& \quad \leq \pi_{q}^{R R}\left|\tilde{x}_{q}^{\tau_{q}}-x_{q}^{\tau_{q}}\right|+\pi_{q}^{R I}\left|\tilde{y}_{q}^{\tau_{q}}-y_{q}^{\tau_{q}}\right| \\
& \left|m_{q}^{I}\left(\tilde{x}_{q}^{\tau_{q}}, \tilde{y}_{q}^{\tau_{q}}\right)-m_{q}^{I}\left(x_{q}^{\tau_{q}}, y_{q}^{\tau_{q}}\right)\right| \\
& \quad \leq \pi_{q}^{I R}\left|\tilde{x}_{q}^{\tau_{q}}-x_{q}^{\tau_{q}}\right|+\pi_{q}^{I I}\left|\tilde{y}_{q}^{\tau_{q}}-y_{q}^{\tau_{q}}\right| .
\end{aligned}
$$

Remark 2. For the dynamic analysis of complex-valued recurrent neural networks models, the most results are based on the existence, continuity, and boundedness of the partial derivatives for real and imaginary parts [32, 33, 46, 49]. Then, by the mean value theorem, it can be proved that they satisfy the inequalities similar to those in Assumption 1. In fact, only the inequalities can be used in the process of obtaining the main results. Thus, the existence, continuity, and boundedness of the partial derivatives are unnecessary and they also lead to limitations in choosing complex-valued activation functions. Here, we remove these constraints and provide a more suitable assumption, Assumption 1, made on the activation functions, so that the current work can be applied to solve more engineering problems. This advantage can be seen from example 2 .

Remark 3. It should be noticed that such an assumption is made on the activation functions in the literature $[44,45]$; i.e., $h_{q}(z)=h_{q}^{R}(\operatorname{Re}(z))+i h_{q}^{I}(\operatorname{Im}(z)), m_{q}(z)=m_{q}^{R}(\operatorname{Re}(z))+$ $i m_{q}^{I}(\operatorname{Im}(z))$ for the complex number $z$ and the following inequalities hold:

$$
\xi_{q}^{R-} \leq \frac{h_{q}^{R}\left(x_{1}\right)-h_{q}^{R}\left(x_{2}\right)}{x_{1}-x_{2}} \leq \xi_{q}^{R+},
$$


Complexity

3

$$
\begin{gathered}
\xi_{q}^{I-} \leq \frac{h_{q}^{I}\left(y_{1}\right)-h_{q}^{I}\left(y_{2}\right)}{y_{1}-y_{2}} \leq \xi_{q}^{I+} \\
\zeta_{q}^{R-} \leq \frac{m_{q}^{R}\left(x_{1}\right)-m_{q}^{R}\left(x_{2}\right)}{x_{1}-x_{2}} \leq \zeta_{q}^{R+}, \\
\zeta_{q}^{I-} \leq \frac{m_{q}^{I}\left(y_{1}\right)-m_{q}^{I}\left(y_{2}\right)}{y_{1}-y_{2}} \leq \zeta_{q}^{I+}
\end{gathered}
$$

for all $q=1,2, \ldots, n$, where $z_{1}=x_{1}+y_{1}$ and $z_{2}=x_{2}+y_{2}$. Obviously, this assumption is a special case of Assumption 1. Therefore, in this paper, we will not discuss this case in detail, but a numerical example for this case will be provided to show the effectiveness.

In this article, the corresponding response system is defined by

$$
\begin{aligned}
\dot{\vec{z}}_{p}(t)= & -d_{p} \widetilde{z}_{p}(t)+\sum_{q=1}^{n} a_{p q} h_{q}\left(\widetilde{z}_{q}(t)\right) \\
& +\sum_{q=1}^{n} b_{p q} m_{q}\left(\widetilde{z}_{q}\left(t-\tau_{q}\right)\right)+J_{p}(t)+u_{p}(t)
\end{aligned}
$$

with the initial conditions

$$
\tilde{z}_{p}(\theta)=\widetilde{\psi}_{p}(\theta), \quad \theta \in[-\tau, 0], p=1,2, \ldots, n .
$$

Let $z_{p}(t)=x_{p}(t)+i y_{p}(t), J_{p}(t)=J_{p}^{R}(t)+i J_{p}^{I}(t), \psi_{p}(s)=$ $\psi_{p}^{R}(s)+i \psi_{p}^{I}(s), a_{p q}=a_{p q}^{R}+i a_{p q}^{I}, b_{p q}=b_{p q}^{R}+i b_{p q}^{I}, \widetilde{z}_{p}(t)=\tilde{x}_{p}(t)+$ $i \tilde{y}_{p}(t), \widetilde{\psi}_{p}(s)=\widetilde{\psi}_{p}^{R}(s)+i \widetilde{\psi}_{p}^{I}(s), h_{q}\left(\widetilde{z}_{q}(t)\right)=h_{q}^{R}\left(\tilde{x}_{q}(t), \widetilde{y}_{q}(t)\right)+$ $i h_{q}^{I}\left(\tilde{x}_{q}(t), \tilde{y}_{q}(t)\right), m_{q}\left(\tilde{z}_{q}\left(t-\tau_{q}\right)\right)=m_{q}^{R}\left(\tilde{x}_{q}\left(t-\tau_{q}\right), \tilde{y}_{q}\left(t-\tau_{q}\right)\right)+$ $i m_{q}^{I}\left(\tilde{x}_{q}\left(t-\tau_{q}\right), \tilde{y}_{q}\left(t-\tau_{q}\right)\right)$, and $u_{p}(t)=u_{p}^{R}(t)+i u_{p}^{I}(t)$. Then, by separating system (1) with (2) and system (5) with (6) into the real and imaginary parts, respectively, we have

$$
\begin{aligned}
\dot{x}_{p}= & -d_{p} x_{p}+\sum_{q=1}^{n} a_{p q}^{R} h_{q}^{R}\left(x_{q}, y_{q}\right) \\
& -\sum_{q=1}^{n} a_{p q}^{I} h_{q}^{I}\left(x_{q}, y_{q}\right)+\sum_{q=1}^{n} b_{p q}^{R} m_{q}^{R}\left(x_{q}^{\tau_{q}}, y_{q}^{\tau_{q}}\right) \\
& -\sum_{q=1}^{n} b_{p q}^{I} m_{q}^{I}\left(x_{q}^{\tau_{q}}, y_{q}^{\tau_{q}}\right)+J_{p}^{R}(t) \\
\dot{y}_{p}= & -d_{p} y_{p}+\sum_{q=1}^{n} a_{p q}^{I} h_{q}^{R}\left(x_{q}, y_{q}\right) \\
& +\sum_{q=1}^{n} a_{p q}^{R} h_{q}^{I}\left(x_{q}, y_{q}\right)+\sum_{q=1}^{n} b_{p q}^{I} m_{q}^{R}\left(x_{q}^{\tau_{q}}, y_{q}^{\tau_{q}}\right) \\
& +\sum_{q=1}^{n} b_{p q}^{R} m_{q}^{I}\left(x_{q}^{\tau_{q}}, y_{q}^{\tau_{q}}\right)+J_{p}^{I}(t)
\end{aligned}
$$

$$
\begin{aligned}
& x_{p}(\theta)=\psi_{p}^{R}(\theta), \\
& y_{p}(\theta)=\psi_{p}^{I}(\theta),
\end{aligned}
$$

$$
\theta \in[-\tau, 0], p=1,2, \ldots, n
$$

and

$$
\begin{aligned}
& \dot{\tilde{x}}_{p}=-d_{p} \tilde{x}_{p}+\sum_{q=1}^{n} a_{p q}^{R} h_{q}^{R}\left(\tilde{x}_{q}, \tilde{y}_{q}\right) \\
&-\sum_{q=1}^{n} a_{p q}^{I} h_{q}^{I}\left(\tilde{x}_{q}, \tilde{y}_{q}\right)+\sum_{q=1}^{n} b_{p q}^{R} m_{q}^{R}\left(\tilde{x}_{q}^{\tau_{q}}, \tilde{y}_{q}^{\tau_{q}}\right) \\
&-\sum_{q=1}^{n} b_{p q}^{I} m_{q}^{I}\left(\tilde{x}_{q}^{\tau_{q}}, \tilde{y}_{q}^{\tau_{q}}\right)+J_{p}^{R}(t)+u_{p}^{R}(t) \\
& \dot{\tilde{y}}_{p}=-d_{p} \tilde{y}_{p}+\sum_{q=1}^{n} a_{p q}^{I} h_{q}^{R}\left(\tilde{x}_{q}, \tilde{y}_{q}\right) \\
&+\sum_{q=1}^{n} a_{p q}^{R} h_{q}^{I}\left(\tilde{x}_{q}, \tilde{y}_{q}\right)+\sum_{q=1}^{n} b_{p q}^{I} m_{q}^{R}\left(\tilde{x}_{q}^{\tau_{q}}, \tilde{y}_{q}^{\tau_{q}}\right) \\
&+\sum_{q=1}^{n} b_{p q}^{R} m_{q}^{I}\left(\tilde{x}_{q}^{\tau_{q}}, \tilde{y}_{q}^{\tau_{q}}\right)+J_{p}^{I}(t)+u_{p}^{I}(t) \\
& \tilde{x}_{p}(\theta)= \widetilde{\varphi}_{p}^{R}(\theta), \\
& \tilde{y}_{p}(\theta)= \widetilde{\varphi}_{p}^{I}(\theta), \\
& \theta \in[-\tau, 0], p=1,2, \ldots, n .
\end{aligned}
$$

Define the error vector between the drive system (1) and the response system (5) as $e_{p}(t)=\widetilde{z}_{p}(t)-z_{p}(t)=e_{p}^{R}(t)+$ $i e_{p}^{I}(t)=\tilde{x}_{p}(t)-x_{p}(t)+i\left(\tilde{y}_{p}(t)-y_{p}(t)\right)$; it follows from $(7)$ and (8) that

$$
\begin{aligned}
\dot{e}_{p}^{R}(t)= & -d_{p} e_{p}^{R}(t)+\sum_{q=1}^{n} a_{p q}^{R}\left(h_{q}^{R}\left(\tilde{x}_{q}, \tilde{y}_{q}\right)-h_{q}^{R}\left(x_{q}, y_{q}\right)\right) \\
& -\sum_{q=1}^{n} a_{p q}^{I}\left(h_{q}^{I}\left(\tilde{x}_{q}, \tilde{y}_{q}\right)-h_{q}^{I}\left(x_{q}, y_{q}\right)\right) \\
& +\sum_{q=1}^{n} b_{p q}^{R}\left(m_{q}^{R}\left(\tilde{x}_{q}^{\tau_{q}}, \tilde{y}_{q}^{\tau_{q}}\right)-m_{q}^{R}\left(x_{q}^{\tau_{q}}, y_{q}^{\tau_{q}}\right)\right) \\
& -\sum_{q=1}^{n} b_{p q}^{I}\left(m_{q}^{I}\left(\tilde{x}_{q}^{\tau_{q}}, \tilde{y}_{q}^{\tau_{q}}\right)-m_{q}^{I}\left(x_{q}^{\tau_{q}}, y_{q}^{\tau_{q}}\right)\right) \\
& +u_{p}^{R}(t) \\
\dot{e}_{p}^{I}(t)= & -d_{p} e_{p}^{I}(t)+\sum_{q=1}^{n} a_{p q}^{I}\left(h_{q}^{R}\left(\tilde{x}_{q}, \tilde{y}_{q}\right)-h_{q}^{R}\left(x_{q}, y_{q}\right)\right)
\end{aligned}
$$




$$
\begin{aligned}
& +\sum_{q=1}^{n} a_{p q}^{R}\left(h_{q}^{I}\left(\tilde{x}_{q}, \tilde{y}_{q}\right)-h_{q}^{I}\left(x_{q}, y_{q}\right)\right) \\
& +\sum_{q=1}^{n} b_{p q}^{I}\left(m_{q}^{R}\left(\tilde{x}_{q}^{\tau_{q}}, \tilde{y}_{q}^{\tau_{q}}\right)-m_{q}^{R}\left(x_{q}^{\tau_{q}}, y_{q}^{\tau_{q}}\right)\right) \\
& +\sum_{q=1}^{n} b_{p q}^{R}\left(m_{q}^{I}\left(\tilde{x}_{q}^{\tau_{q}}, \tilde{y}_{q}^{\tau_{q}}\right)-m_{q}^{I}\left(x_{q}^{\tau_{q}}, y_{q}^{\tau_{q}}\right)\right) \\
& +u_{p}^{I}(t) \\
e_{p}^{R}(\theta)= & \phi_{p}^{R}(\theta), \\
e_{p}^{I}(\theta)= & \phi_{p}^{I}(\theta),
\end{aligned}
$$

$$
\theta \in[-\tau, 0], p=1,2, \ldots, n \text {. }
$$

Let $\omega_{p}(t)=\left[\begin{array}{c}e_{p}^{R}(t) \\ e_{p}^{I}(t)\end{array}\right]$; then equation (9) can be rewritten as

$$
\begin{aligned}
\dot{\omega}_{p}(t) & =-\left[\begin{array}{cc}
d_{p} & 0 \\
0 & d_{p}
\end{array}\right] \omega_{p}(t) \\
+ & \sum_{q=1}^{n}\left[\begin{array}{cc}
a_{p q}^{R} & -a_{p q}^{I} \\
a_{p q}^{I} & a_{p q}^{R}
\end{array}\right]\left[\begin{array}{c}
h_{q}^{R}\left(\tilde{x}_{q}, \tilde{y}_{q}\right)-h_{q}^{R}\left(x_{q}, y_{q}\right) \\
h_{q}^{I}\left(\tilde{x}_{q}, \tilde{y}_{q}\right)-h_{q}^{I}\left(x_{q}, y_{q}\right)
\end{array}\right] \\
+ & \sum_{q=1}^{n}\left[\begin{array}{cc}
b_{p q}^{R} & -b_{p q}^{I} \\
b_{p q}^{I} & b_{p q}^{R}
\end{array}\right]\left[\begin{array}{l}
m_{q}^{R}\left(\tilde{x}_{q}^{\tau_{q}}, \tilde{y}_{q}^{\tau_{q}}\right)-m_{q}^{R}\left(x_{q}^{\tau_{q}}, y_{q}^{\tau_{q}}\right) \\
m_{q}^{I}\left(\tilde{x}_{q}^{\tau_{q}}, \tilde{y}_{q}^{\tau_{q}}\right)-m_{q}^{I}\left(x_{q}^{\tau_{q}}, y_{q}^{\tau_{q}}\right)
\end{array}\right] \\
+ & {\left[\begin{array}{c}
u_{p}^{R}(t) \\
u_{p}^{I}(t)
\end{array}\right] }
\end{aligned}
$$$$
\omega_{p}(\theta)=\phi_{p}(\theta)=\left[\begin{array}{l}
\phi_{p}^{R}(\theta) \\
\phi_{p}^{I}(\theta)
\end{array}\right], \quad \theta \in[-\tau, 0]
$$

for $p=1,2, \ldots, n$.

Before deriving the main results, the following definition and lemmas are provided.

Definition 4 (see [27]). Consider the drive-response systems (1) with (2) and (5) with (6). If for a suitable controller $u_{p}(t)=$ $u_{p}^{R}(t)+i u_{p}^{I}(t)$, there exists a function $T=T\left(\phi_{p}\right)>0$ depending on the initial value $\phi_{p}$, such that

$$
\lim _{t \longrightarrow T}\left|e_{p}^{R}(t)\right|=\lim _{t \longrightarrow T}\left|e_{p}^{I}(t)\right|=0
$$

and $\left|e_{p}^{R}(t)\right|=\left|e_{p}^{I}(t)\right|=0$ for $t>T, p=1,2, \ldots, n$, then the drive system (1) and the response system (5) achieve synchronization in finite time.

Lemma 5 (see [24]). An open set $U \in C\left([-\tau, 0], \mathbb{R}^{2 n}\right)$. The class $\mathscr{G}$ denotes all the strictly increasing continuous functions $s: \mathbb{R}_{+} \longrightarrow \mathbb{R}_{+}$and $s(0)=0$. If there exist $s, \varrho \in \mathscr{G}$ and $a$ continuous function $V:[0,+\infty) \times U \longrightarrow \mathbb{R}_{+}$for system (10) such that
(1) $V(t, 0)=0, s(\|x\|) \leq V(t, x), t \in[0,+\infty)$,

(2) $D^{+} V(t, x) \leq-\varrho(V(t, x))$ with $\int_{0}^{\epsilon}(d z / \varrho(z))<+\infty$,

for all $\epsilon>0, x \in U$, then, system (10) is finite-time stable and the settling time $T \leq \int_{0}^{V(0, \phi)}(d z / \varrho(z))$. In particular, if $\varrho(V)=$ $k V^{\mu}(k>0,0<\mu<1)$, then the settling time

$$
T \leq \int_{0}^{V(0, \phi)} \frac{d z}{\varrho(z)}=\frac{V^{1-\mu}(0, \phi)}{k(1-\mu)} .
$$

Lemma 6 (see [52]). If $a>0, b>0,0<\alpha_{1}<1,0<\alpha_{2}<1$, then there must exist a scalar $0<\alpha<1$ depending on $\alpha_{1}$ and $\alpha_{2}$, such that

$$
a^{\alpha_{1}}+b^{\alpha_{2}}>\frac{(a+b)^{\alpha}}{2^{\alpha}} .
$$

Remark 7. In order to deal with the finite-time stabilization problem for complex-valued neural networks system and estimate the upper bound of Dini-derivative of Lyapunov function, the inequality in Lemma 6 was proposed and proved by us in Lemma 8 of [52]. Moreover, from the proof of the inequality stated in [52], we can see that either $\alpha=\alpha_{1}$ or $\alpha=\alpha_{2}$, which can be used to compute the settling time. This point can be shown in examples 1 and 2.

Lemma 8 (see [24]). For real numbers $c_{1}, c_{2}, \ldots, c_{n}$ and $0<$ $k_{1}<k_{2}$, the following inequality holds:

$$
\left[\sum_{q=1}^{n}\left(\left|c_{q}\right|\right)^{k_{2}}\right]^{1 / k_{2}} \leq\left[\sum_{q=1}^{n}\left(\left|c_{q}\right|\right)^{k_{1}}\right]^{1 / k_{1}} .
$$

\section{Main Result}

In this section, new sufficient conditions will be derived to ascertain the finite-time synchronization for system (1) and (5). The new nonlinear controller $u_{p}(t)$ in response system (5) is designed as the following form:

$$
\begin{aligned}
u_{p}(t)= & u_{p}^{R}(t)+i u_{p}^{I}(t) \\
u_{p}^{R}(t)= & -\alpha_{1 p} e_{p}^{R}(t)-\beta_{1 p}\left|e_{p}^{R}(t)\right|^{\mu_{1}} \operatorname{sgn}\left(e_{p}^{R}(t)\right) \\
& -\gamma_{1 p} \sum_{q=1}^{n}\left|e_{q}^{R}\left(t-\tau_{q}\right)\right| \operatorname{sgn}\left(e_{p}^{R}(t)\right) \\
u_{p}^{I}(t)= & -\alpha_{2 p} e_{p}^{I}(t)-\beta_{2 p}\left|e_{p}^{I}(t)\right|^{\mu_{2}} \operatorname{sgn}\left(e_{p}^{I}(t)\right) \\
& -\gamma_{2 p} \sum_{q=1}^{n}\left|e_{q}^{I}\left(t-\tau_{q}\right)\right| \operatorname{sgn}\left(e_{p}^{I}(t)\right)
\end{aligned}
$$

where $\alpha_{1 p}, \alpha_{2 p}, \beta_{1 p}>0, \beta_{2 p}>0, \gamma_{1 p}, \gamma_{2 p}, 0<\mu_{1}<1$, and $0<\mu_{2}<1$ are constants for $p=1,2, \ldots, n$.

Remark 9. In this paper, we design the controller (15) with independent parameters $\mu_{1}$ and $\mu_{2}$, which are different from 
the most existing results based on $\mu_{1}=\mu_{2}$, such as $[17,18,22]$. This is one of the main features for our work, which also leads to difficulty in studying finite-time dynamic behavior of the considered system. To solve this difficulty, we use the inequality in Lemma 6 proposed and proved by us in [52].

First, for convenience, we denote

$$
\begin{aligned}
& \kappa^{R R}=\operatorname{diag}\left\{\kappa_{1}^{R R}, \kappa_{2}^{R R}, \ldots, \kappa_{n}^{R R}\right\}, \\
& \kappa^{R I}=\operatorname{diag}\left\{\kappa_{1}^{R I}, \kappa_{2}^{R I}, \ldots, \kappa_{n}^{R I}\right\}, \\
& A^{R}=\left(\left|a_{p q}^{R}\right|\right)_{n \times n}, \\
& \kappa^{I R}=\operatorname{diag}\left\{\kappa_{1}^{I R}, \kappa_{2}^{I R}, \ldots, \kappa_{n}^{I R}\right\}, \\
& \kappa^{I I}=\operatorname{diag}\left\{\kappa_{1}^{I I}, \kappa_{2}^{I I}, \ldots, \kappa_{n}^{I I}\right\}, \\
& A^{I}=\left(\left|a_{p q}^{I}\right|\right)_{n \times n}, \\
& \pi^{R R}=\operatorname{diag}\left\{\pi_{1}^{R R}, \pi_{2}^{R R}, \ldots, \pi_{n}^{R R}\right\}, \\
& \pi^{R I}=\operatorname{diag}\left\{\pi_{1}^{R I}, \pi_{2}^{R I}, \ldots, \pi_{n}^{R I}\right\}, \\
& B^{R}=\left(\left|b_{p q}^{R}\right|\right)_{n \times n}, \\
& \pi^{I R}=\operatorname{diag}\left\{\pi_{1}^{I R}, \pi_{2}^{I R}, \ldots, \pi_{n}^{I R}\right\}, \\
& \pi^{I I}=\operatorname{diag}\left\{\pi_{1}^{I I}, \pi_{2}^{I I}, \ldots, \pi_{n}^{I I}\right\}, \\
& B^{I}=\left(\left|b_{p q}^{I}\right|\right)_{n \times n}, \\
& \Gamma_{1}=\operatorname{diag}\left\{\gamma_{11}, \gamma_{12}, \ldots, \gamma_{1 n}\right\}, \\
& \bar{\Gamma}_{1}=\operatorname{tr}\left(\Gamma_{1}\right) E_{n}, \\
& \Gamma_{2}=\operatorname{diag}\left\{\gamma_{21}, \gamma_{22}, \ldots, \gamma_{2 n}\right\} \text {, } \\
& \bar{\Gamma}_{2}=\operatorname{tr}\left(\Gamma_{2}\right) E_{n}, \\
& E_{n}=\operatorname{diag}\{1,1, \ldots, 1\} \text {, } \\
& D_{1}=\operatorname{diag}\left\{d_{1}+\alpha_{11}, d_{2}+\alpha_{12}, \ldots, d_{n}+\alpha_{1 n}\right\} \text {, } \\
& D_{2}=\operatorname{diag}\left\{d_{1}+\alpha_{21}, d_{2}+\alpha_{22}, \ldots, d_{n}+\alpha_{2 n}\right\} \text {, } \\
& I_{n}=[1,1, \ldots, 1]^{T} \text {, } \\
& I=\operatorname{col}\left\{I_{n}, I_{n}\right\}, \\
& D=\left[\begin{array}{cc}
D_{1} & 0 \\
0 & D_{2}
\end{array}\right] \text {, } \\
& \bar{\Gamma}=\left[\begin{array}{cc}
\bar{\Gamma}_{1} & 0 \\
0 & \bar{\Gamma}_{2}
\end{array}\right] \text {, } \\
& A=\left[\begin{array}{ll}
A^{R} & A^{I} \\
A^{I} & A^{R}
\end{array}\right],
\end{aligned}
$$

$$
\begin{aligned}
B & =\left[\begin{array}{ll}
B^{R} & B^{I} \\
B^{I} & B^{R}
\end{array}\right], \\
\kappa & =\left[\begin{array}{ll}
\kappa^{R R} & \kappa^{R I} \\
\kappa^{I R} & \kappa^{I I}
\end{array}\right], \\
\pi & =\left[\begin{array}{ll}
\pi^{R R} & \pi^{R I} \\
\pi^{I R} & \pi^{I I}
\end{array}\right], \\
G_{1} & =D-(A \kappa)^{T}, \\
G_{2} & =\bar{\Gamma}-(B \pi)^{T} .
\end{aligned}
$$

Based on the controller (15), the main result of our paper can be obtained as follows.

Theorem 10. Under Assumption 1, if the vectors $G_{1} I>0$ and $G_{2} I>0$, then via the controller (15), the drive system (1) and the response system (5) are synchronized in finite time. Moreover, the settling time satisfies $T_{2} \leq(2 / \beta(1-\mu)) V^{1-\mu}(0)$, where $\beta=$ $\min \left\{\beta_{1}, \beta_{2}\right\}, \beta_{1}=\min \left\{\beta_{1 p}\right\}, \beta_{2}=\min \left\{\beta_{2 p}\right\}, p=1,2, \ldots, n$, and $0<\mu<1$ depending on $\mu_{1}$ and $\mu_{2}$.

Proof. If $G_{1} I>0$ holds, then for all $p=1,2, \ldots, n$, we have

$$
\begin{aligned}
d_{p} & +\alpha_{1 p} \\
& -\sum_{q=1}^{n}\left(\left|a_{q p}^{R}\right| \kappa_{p}^{R R}+\left|a_{q p}^{I}\right| \kappa_{p}^{I R}+\left|a_{q p}^{I}\right| \kappa_{p}^{R R}+\left|a_{q p}^{R}\right| \kappa_{p}^{I R}\right) \\
& >0 \\
d_{p} & +\alpha_{2 p} \\
& -\sum_{q=1}^{n}\left(\left|a_{q p}^{I}\right| \kappa_{p}^{R I}+\left|a_{q p}^{R}\right| \kappa_{p}^{I I}+\left|a_{q p}^{R}\right| \kappa_{p}^{R I}+\left|a_{q p}^{I}\right| \kappa_{p}^{I I}\right) \\
> & 0 .
\end{aligned}
$$

Similarly, if $G_{2} I>0$ holds, then for all $p=1,2, \ldots, n$, we have

$$
\begin{aligned}
& \sum_{q=1}^{n} \gamma_{1 q} \\
& \quad-\sum_{q=1}^{n}\left(\left|b_{q p}^{R}\right| \pi_{p}^{R R}+\left|b_{q p}^{I}\right| \pi_{p}^{I R}+\left|b_{q p}^{I}\right| \pi_{p}^{R R}+\left|b_{q p}^{R}\right| \pi_{p}^{I R}\right) \\
& \quad>0 \\
& \sum_{q=1}^{n} \gamma_{2 q} \\
& \quad-\sum_{q=1}^{n}\left(\left|b_{q p}^{R}\right| \pi_{p}^{R I}+\left|b_{q p}^{I}\right| \pi_{p}^{I I}+\left|b_{q p}^{I}\right| \pi_{p}^{R I}+\left|b_{q p}^{R}\right| \pi_{p}^{I I}\right) \\
& \quad>0 .
\end{aligned}
$$


6

Complexity

For the error system (9) or (10), choose the following Lyapunov function:

$$
V(t)=\sum_{p=1}^{n}\left|e_{p}^{R}(t)\right|+\sum_{p=1}^{n}\left|e_{p}^{I}(t)\right| .
$$

Along the trajectories of system (9) or (10) and by (15), the upper Dini-derivative of $V(t)$ can be computed as

$$
\begin{aligned}
& D^{+} V(t)=\sum_{p=1}^{n} \operatorname{sgn}\left(e_{p}^{R}(t)\right) \dot{e}_{p}^{R}(t) \\
& +\sum_{p=1}^{n} \operatorname{sgn}\left(e_{p}^{I}(t)\right) \dot{e}_{p}^{I}(t)=\sum_{p=1}^{n} \operatorname{sgn}\left(e_{p}^{R}(t)\right) \\
& \cdot\left[-d_{p} e_{p}^{R}(t)+\sum_{q=1}^{n} a_{p q}^{R}\left(h_{q}^{R}\left(\tilde{x}_{q}, \tilde{y}_{q}\right)-h_{q}^{R}\left(x_{q}, y_{q}\right)\right)\right. \\
& -\sum_{q=1}^{n} a_{p q}^{I}\left(h_{q}^{I}\left(\tilde{x}_{q}, \tilde{y}_{q}\right)-h_{q}^{I}\left(x_{q}, y_{q}\right)\right) \\
& +\sum_{q=1}^{n} b_{p q}^{R}\left(m_{q}^{R}\left(\tilde{x}_{q}^{\tau_{q}}, \tilde{y}_{q}^{\tau_{q}}\right)-m_{q}^{R}\left(x_{q}^{\tau_{q}}, y_{q}^{\tau_{q}}\right)\right) \\
& -\sum_{q=1}^{n} b_{p q}^{I}\left(m_{q}^{I}\left(\tilde{x}_{q}^{\tau_{q}}, \tilde{y}_{q}^{\tau_{q}}\right)-m_{q}^{I}\left(x_{q}^{\tau_{q}}, y_{q}^{\tau_{q}}\right)\right) \\
& -\alpha_{1 p} e_{p}^{R}(t)-\beta_{1 p}\left|e_{p}^{R}(t)\right|^{\mu_{1}} \operatorname{sgn}\left(e_{p}^{R}(t)\right) \\
& \left.-\gamma_{1 p} \sum_{q=1}^{n}\left|e_{q}^{R}\left(t-\tau_{q}\right)\right| \operatorname{sgn}\left(e_{p}^{R}(t)\right)\right] \\
& +\sum_{p=1}^{n} \operatorname{sgn}\left(e_{p}^{I}(t)\right)\left[-d_{p} e_{p}^{I}(t)\right. \\
& +\sum_{q=1}^{n} a_{p q}^{I}\left(h_{q}^{R}\left(\tilde{x}_{q}, \tilde{y}_{q}\right)-h_{q}^{R}\left(x_{q}, y_{q}\right)\right) \\
& +\sum_{q=1}^{n} a_{p q}^{R}\left(h_{q}^{I}\left(\tilde{x}_{q}, \tilde{y}_{q}\right)-h_{q}^{I}\left(x_{q}, y_{q}\right)\right) \\
& +\sum_{q=1}^{n} b_{p q}^{I}\left(m_{q}^{R}\left(\tilde{x}_{q}^{\tau_{q}}, \tilde{y}_{q}^{\tau_{q}}\right)-m_{q}^{R}\left(x_{q}^{\tau_{q}}, y_{q}^{\tau_{q}}\right)\right) \\
& +\sum_{q=1}^{n} b_{p q}^{R}\left(m_{q}^{I}\left(\tilde{x}_{q}^{\tau_{q}}, \tilde{y}_{q}^{\tau_{q}}\right)-m_{q}^{I}\left(x_{q}^{\tau_{q}}, y_{q}^{\tau_{q}}\right)\right) \\
& -\alpha_{2 p} e_{p}^{I}(t)-\beta_{2 p}\left|e_{p}^{I}(t)\right|^{\mu_{2}} \operatorname{sgn}\left(e_{p}^{I}(t)\right) \\
& \left.-\gamma_{2 p} \sum_{q=1}^{n}\left|e_{q}^{I}\left(t-\tau_{q}\right)\right| \operatorname{sgn}\left(e_{p}^{I}(t)\right)\right] \text {. }
\end{aligned}
$$

$$
\begin{aligned}
D^{+} & V(t) \leq \sum_{p=1}^{n}\left[-\left(d_{p}+\alpha_{1 p}\right)\left|e_{p}^{R}(t)\right|\right. \\
& +\sum_{q=1}^{n}\left|a_{p q}^{R}\right|\left(\kappa_{q}^{R R}\left|e_{q}^{R}(t)\right|+\kappa_{q}^{R I}\left|e_{q}^{I}(t)\right|\right) \\
& +\sum_{q=1}^{n}\left|a_{p q}^{I}\right|\left(\kappa_{q}^{I R}\left|e_{q}^{R}(t)\right|+\kappa_{q}^{I I}\left|e_{q}^{I}(t)\right|\right) \\
& +\sum_{q=1}^{n}\left|b_{p q}^{R}\right|\left(\pi_{q}^{R R}\left|e_{q}^{R}\left(t-\tau_{q}\right)\right|+\pi_{q}^{R I}\left|e_{q}^{I}\left(t-\tau_{q}\right)\right|\right) \\
& +\sum_{q=1}^{n}\left|b_{p q}^{I}\right|\left(\pi_{q}^{I R}\left|e_{q}^{R}\left(t-\tau_{q}\right)\right|+\pi_{q}^{I I}\left|e_{q}^{I}\left(t-\tau_{q}\right)\right|\right) \\
& \left.+\beta_{2 p}\left|e_{p}^{I}(t)\right|^{\mu_{2}}-\gamma_{2 p} \sum_{q=1}^{n}\left|e_{q}^{I}\left(t-\tau_{q}\right)\right|\right] \\
& +\sum_{1 p}\left|e_{p}^{R}(t)\right| b_{p q}^{R} \mid\left(\pi_{q}^{I R}\left|e_{q}^{R}\left(t-\tau_{q}\right)\right|+\pi_{q}^{I I}\left|e_{q}^{I}\left(t-\tau_{q}\right)\right|\right) \\
& \left.+\sum_{q=1}^{\mu_{1}}\left|a_{q=1}^{n}\right| e_{q}^{R}\left(t-\tau_{q}\right) \mid\right] \\
& +\sum_{p=1}^{n} \mid-\left(\kappa_{q}^{I R}\left|e_{q}^{R}(t)\right|+\kappa_{q}^{I I}\left|e_{q}^{I}(t)\right|\right) \\
& +a_{p q}^{I} \mid\left(\kappa_{q}^{R R}\left|e_{q}^{R}(t)\right|+\kappa_{q}^{R I}\left|e_{q}^{I}(t)\right|\right)
\end{aligned}
$$

By uniting like terms, we have

$$
\begin{aligned}
& D^{+} V(t) \leq \sum_{p=1}^{n}\left[-\left(d_{p}+\alpha_{1 p}\right)\left|e_{p}^{R}(t)\right|\right. \\
& +\sum_{q=1}^{n}\left(\left|a_{p q}^{R}\right| \kappa_{q}^{R R}+\left|a_{p q}^{I}\right| \kappa_{q}^{I R}\right)\left|e_{q}^{R}(t)\right| \\
& +\sum_{q=1}^{n}\left(\left|a_{p q}^{R}\right| \kappa_{q}^{R I}+\left|a_{p q}^{I}\right| \kappa_{q}^{I I}\right)\left|e_{q}^{I}(t)\right| \\
& +\sum_{q=1}^{n}\left(\left|b_{p q}^{R}\right| \pi_{q}^{R R}+\left|b_{p q}^{I}\right| \pi_{q}^{I R}-\gamma_{1 p}\right)\left|e_{q}^{R}\left(t-\tau_{q}\right)\right| \\
& +\sum_{q=1}^{n}\left(\left|b_{p q}^{R}\right| \pi_{q}^{R I}+\left|b_{p q}^{I}\right| \pi_{q}^{I I}\right)\left|e_{q}^{I}\left(t-\tau_{q}\right)\right|
\end{aligned}
$$




$$
\begin{aligned}
& \left.-\beta_{1 p}\left|e_{p}^{R}(t)\right|^{\mu_{1}}\right]+\sum_{p=1}^{n}\left[-\left(d_{p}+\alpha_{2 p}\right)\left|e_{p}^{I}(t)\right|\right. \\
& +\sum_{q=1}^{n}\left(\left|a_{p q}^{I}\right| \kappa_{q}^{R R}+\left|a_{p q}^{R}\right| \kappa_{q}^{I R}\right)\left|e_{q}^{R}(t)\right| \\
& +\sum_{q=1}^{n}\left(\left|a_{p q}^{I}\right| \kappa_{q}^{R I}+\left|a_{p q}^{R}\right| \kappa_{q}^{I I}\right)\left|e_{q}^{I}(t)\right| \\
& \left.+\sum_{q=1}^{n}\left(\left|b_{p q}^{I}\right| \pi_{q}^{R I}+\left|b_{p q}^{R}\right| \pi_{q}^{I I}-\gamma_{2 p}\right)\left|e_{q}^{I}\left(t-\tau_{q}\right)\right|\right] \\
& +\sum_{q=1}^{n}\left(\left|b_{p q}^{I}\right| \pi_{q}^{R R}+\left|b_{p q}^{R}\right| \pi_{q}^{I R}\right)\left|e_{q}^{R}\left(t-\tau_{q}\right)\right| \\
& -\beta_{2 p}\left|e_{p}^{I}(t)\right|^{\mu_{2}} .
\end{aligned}
$$

Then, by taking the common factors, one has

$$
\begin{aligned}
& D^{+} V(t) \leq \sum_{p=1}^{n}\left|e_{p}^{R}(t)\right|\left[-\left(d_{p}+\alpha_{1 p}\right)\right. \\
& \left.\quad+\sum_{q=1}^{n}\left(\left|a_{q p}^{R}\right| \kappa_{p}^{R R}+\left|a_{q p}^{I}\right| \kappa_{p}^{I R}+\left|a_{q p}^{I}\right| \kappa_{p}^{R R}+\left|a_{q p}^{R}\right| \kappa_{p}^{I R}\right)\right] \\
& \quad+\sum_{p=1}^{n}\left|e_{p}^{I}(t)\right|\left[-\left(d_{p}+\alpha_{2 p}\right)\right. \\
& \left.+\sum_{q=1}^{n}\left(\left|a_{q p}^{I}\right| \kappa_{p}^{R I}+\left|a_{q p}^{R}\right| \kappa_{p}^{I I}+\left|a_{q p}^{R}\right| \kappa_{p}^{R I}+\left|a_{q p}^{I}\right| \kappa_{p}^{I I}\right)\right] \\
& \quad+\sum_{p=1}^{n} \sum_{q=1}^{n}\left(\left|b_{q p}^{R}\right| \pi_{p}^{R R}+\left|b_{q p}^{I}\right| \pi_{p}^{I R}-\gamma_{1 q}+\left|b_{q p}^{I}\right| \pi_{p}^{R R}\right. \\
& \left.\quad+\left|b_{q p}^{R}\right| \pi_{p}^{I R}\right)\left|e_{p}^{R}\left(t-\tau_{p}\right)\right|+\sum_{p=1}^{n} \sum_{q=1}^{n}\left(\left|b_{q p}^{R}\right| \pi_{p}^{R I}\right. \\
& \left.\quad+\left|b_{q p}^{I}\right| \pi_{p}^{I I}+\left|b_{q p}^{I}\right| \pi_{p}^{R I}+\left|b_{q p}^{R}\right| \pi_{p}^{I I}-\gamma_{2 q}\right)\left|e_{p}^{I}\left(t-\tau_{p}\right)\right| \\
& \quad-\sum_{p=1}^{n} \beta_{1 p}\left|e_{p}^{R}(t)\right|^{\mu_{1}}-\sum_{p=1}^{n} \beta_{2 p}\left|e_{p}^{I}(t)\right|^{\mu_{2}} .
\end{aligned}
$$

Then, by (17) and (18), one can obtain

$$
\begin{aligned}
D^{+} V(t) & \leq-\sum_{\mathrm{p}=1}^{n} \beta_{1 p}\left|e_{p}^{R}(t)\right|^{\mu_{1}}-\sum_{p=1}^{n} \beta_{2 p}\left|e_{p}^{I}(t)\right|^{\mu_{2}} \\
& \leq-\beta_{1} \sum_{p=1}^{n}\left|e_{p}^{R}(t)\right|^{\mu_{1}}-\beta_{2} \sum_{p=1}^{n}\left|e_{p}^{I}(t)\right|^{\mu_{2}} \\
& \leq-\beta\left(\sum_{p=1}^{n}\left|e_{p}^{R}(t)\right|^{\mu_{1}}+\sum_{p=1}^{n}\left|e_{p}^{I}(t)\right|^{\mu_{2}}\right)
\end{aligned}
$$

where $\beta=\min \left\{\beta_{1}, \beta_{2}\right\}, \beta_{1}=\min \left\{\beta_{1 p}\right\}, \beta_{2}=\min \left\{\beta_{2 p}\right\}, p=$ $1,2, \ldots, n$. In addition, by Lemma 8 , for $0<\mu_{1}<1,0<\mu_{2}<$ 1 , the following inequalities hold:

$$
\begin{aligned}
& -\sum_{p=1}^{n}\left|e_{p}^{R}(t)\right|^{\mu_{1}} \leq-\left[\sum_{p=1}^{n}\left|e_{p}^{R}(t)\right|\right]^{\mu_{1}}, \\
& -\sum_{p=1}^{n}\left|e_{p}^{I}(t)\right|^{\mu_{2}} \leq-\left[\sum_{p=1}^{n}\left|e_{p}^{I}(t)\right|\right]^{\mu_{2}} .
\end{aligned}
$$

Then, substituting (25) into (24), one has

$$
D^{+} V(t) \leq-\beta\left(\left[\sum_{p=1}^{n}\left|e_{p}^{R}(t)\right|\right]^{\mu_{1}}+\left[\sum_{p=1}^{n}\left|e_{p}^{I}(t)\right|\right]^{\mu_{2}}\right) .
$$

By Lemma 6, there exists a $0<\mu<1$ depending on $\mu_{1}$ and $\mu_{2}$, such that

$$
\begin{aligned}
D^{+} V(t) & \leq-\frac{\beta}{2^{\mu}}\left(\sum_{p=1}^{n}\left|e_{p}^{R}(t)\right|+\sum_{p=1}^{n}\left|e_{p}^{I}(t)\right|\right)^{\mu} \\
& \leq-\frac{\beta}{2} V^{\mu}(t) .
\end{aligned}
$$

Moreover, $\int_{0}^{\varepsilon}\left(2 / \beta z^{\mu}\right) d z=2 \varepsilon^{1-\mu} / \beta(1-\mu)<+\infty$, for all $\varepsilon>0$. Thus, from Lemma 5 , we know that the error system (9) or (10) is finite-time stable. That is to say, the drive system (1) and the response system (5) can be synchronized in finite time via the controller (15). This completes the proof.

Remark 11. Recently, the finite-time stabilization and synchronization problems of real-valued neural networks have been investigated and the time for system trajectories tending to an equilibrium point is finite [22-29]. However, for finite-time stability analysis of complex-valued neural networks models, until now, few results have been developed. For instance, the finite-time synchronization problem for complex-valued neural networks (CVNNs) with infinitetime distributed delays is discussed in [51] and the finitetime stabilizability and instabilizability for complex-valued memristive neural networks with time delays are studied in [52]. Here, Theorem 10 further provides sufficient conditions for the finite-time synchronization of the considered system and their effectiveness can be illustrated in numerical examples. From the point of theory development, our result is significant in dynamical behavior analysis of complex-valued models and it can be regarded as the extension of the previous work.

\section{Numerical Examples}

This section will provide two examples to show the effectiveness of the given conditions of Theorem 10. 

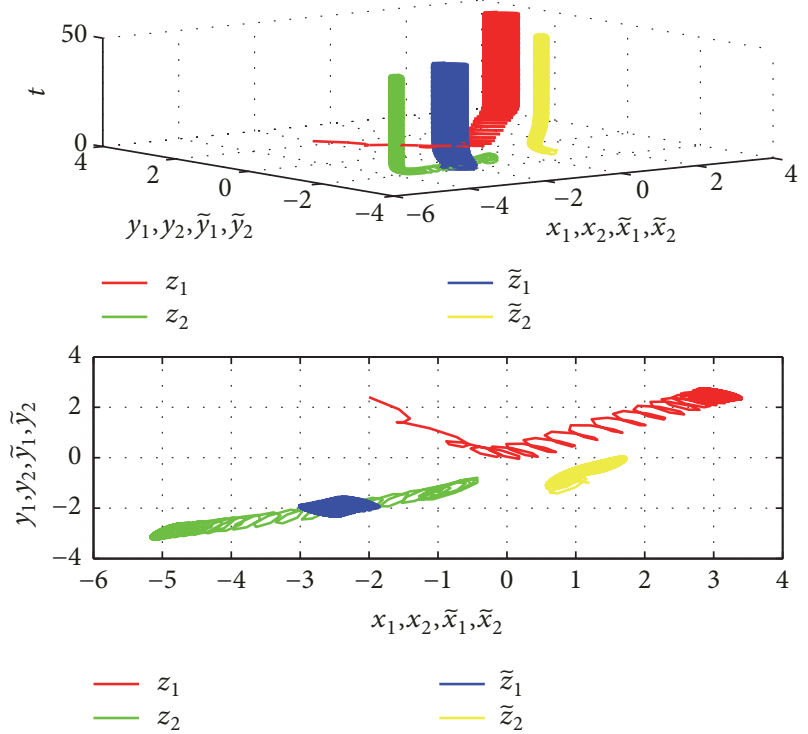

FIGURE 1: Time responses of the variables $z_{1}, z_{2}, \widetilde{z}_{1}$, and $\widetilde{z}_{2}$ in $3 \mathrm{D}$ and 2D spaces without controller for example 1 .

Example 12. Consider complex-valued neural networks as the drive system given by

$$
\begin{aligned}
\dot{z}_{p}(t)= & -d_{p} z_{p}(t)+\sum_{q=1}^{2} a_{p q} h_{q}\left(z_{q}(t)\right) \\
& +\sum_{q=1}^{2} b_{p q} m_{q}\left(z_{q}\left(t-\tau_{q}\right)\right)+J_{p}(t), \quad p=1,2
\end{aligned}
$$

where $d_{1}=1.5, d_{2}=1, a_{11}=1+2 i, a_{12}=-2-i, a_{21}=-2.5-i$, $a_{22}=-1-0.5 i, b_{11}=2-i, b_{12}=-1+i, b_{21}=-1+2 i, b_{22}=2+i$, $\tau_{1}=0.1, \tau_{2}=0.2, J_{1}(t)=6 \sin (t+1)-4 \cos (t-1) i$, and $J_{2}(t)=5 \cos (t+1)-6 \sin (t-1) i$. Moreover, the activation functions are taken as

$$
\begin{aligned}
& h_{q}\left(z_{q}\right)=\frac{1-e^{-x_{q}}}{1+e^{-x_{q}}}+i \frac{1}{1+e^{-y_{q}}}, \\
& m_{q}\left(z_{q}\right)=\frac{1-e^{-y_{q}}}{1+e^{-y_{q}}}+i \frac{1}{1+e^{-x_{q}}},
\end{aligned}
$$

$$
(q=1,2) .
$$

Then, from Assumption 1, it can be computed that $\kappa_{q}^{R R}=0.5$, $\kappa_{q}^{I I}=0.25, \kappa_{q}^{R I}=\kappa_{q}^{I R}=0, \pi_{q}^{R I}=0.5, \pi_{q}^{I R}=0.25$, and $\pi_{q}^{R R}=$ $\pi_{q}^{I I}=0$ for $q=1,2$.

The response system is provided by

$$
\begin{aligned}
\dot{\vec{z}}_{p}(t)= & -d_{p} \widetilde{z}_{p}(t)+\sum_{q=1}^{2} a_{p q} h_{q}\left(\widetilde{z}_{q}(t)\right) \\
& +\sum_{q=1}^{2} b_{p q} m_{q}\left(\widetilde{z}_{q}\left(t-\tau_{q}\right)\right)+J_{p}(t)+u_{p}(t) .
\end{aligned}
$$

Under the initial conditions $z_{1}(\theta)=-2+2.4 i, z_{2}(\theta)=$ $-1-1.4 i, \widetilde{z}_{1}(\theta)=-2.3-1.8 i$, and $\widetilde{z}_{2}(\theta)=1.2-i$ for $\theta \in[-0.2,0]$, Figures 1 and 2 depict the trajectories of systems (28) and (30) without controller, which show that the drive system (28) does not synchronize with the response system (30).

By choosing the parameters of the controller (15) as $\alpha_{11}=$ 2.3, $\alpha_{12}=1.6, \alpha_{21}=1.1, \alpha_{22}=0.8, \beta_{11}=\beta_{12}=\beta_{21}=$ $\beta_{22}=1, \gamma_{11}=1, \gamma_{12}=0.6, \gamma_{21}=1.5, \gamma_{22}=1.6, \mu_{1}=$ 0.5 , and $\mu_{2}=0.6$, it is easy to check that the conditions of Theorem 10 are satisfied. Then, under the corresponding controller, the drive system (28) can be synchronized with the response system (30) in finite time. In addition, according to Theorem 10 and Remark 7, it can be computed that $T_{1} \leq$ $\max \{10.88,10.95\}=10.95$ seconds. The synchronization curves between the drive system (28) and the response system (30) are displayed in Figure 3. The time responses of the synchronization errors between them are shown in Figure 4. From Figures 3 and 4, it can be seen that system (28) can synchronize with system (30) in finite time via the corresponding controller under the given initial values. These also further show that the obtained result is effective and our work is meaningful.

Next, one example with special activation functions stated as in Remarks 2 and 3 is given to illustrate the validity of our result.

Example 13. Consider a drive system as follows:

$$
\begin{aligned}
\dot{z}_{p}(t)= & -d_{p} z_{p}(t)+\sum_{q=1}^{2} a_{p q} h_{q}\left(z_{q}(t)\right) \\
& +\sum_{q=1}^{2} b_{p q} m_{q}\left(z_{q}\left(t-\tau_{q}\right)\right)+J_{p}(t), p=1,2
\end{aligned}
$$

where $d_{1}=1, d_{2}=0.5, a_{11}=-0.5+i, a_{12}=2-i, a_{21}=$ $1.5-1.8 i, a_{22}=-1+2 i, b_{11}=-0.8-0.5 i, b_{12}=-1+1.5 i$, $b_{21}=2+1.5 i, b_{22}=-1+2 i, \tau_{1}=0.1, \tau_{2}=0.2, J_{1}(t)=3-i$, and $J_{2}(t)=-2+5 i$. The activation functions are chosen as

$$
\begin{aligned}
& h_{q}\left(z_{q}\right)=m_{q}\left(z_{q}\right) \\
&=\frac{\left|x_{q}+1\right|-\left|x_{q}-1\right|}{2}+i \frac{\left|y_{q}+1\right|-\left|y_{q}-1\right|}{2}, \\
&(q=1,2) .
\end{aligned}
$$

Obviously, $\left.\left(\partial / \partial x_{q}\right)\left(\left(\left|x_{q}+1\right|-\left|x_{q}-1\right|\right) / 2\right)\right|_{x_{q}= \pm 1}$ and $(\partial /$ $\left.\partial y_{q}\right)\left.\left(\left(\left|y_{q}+1\right|-\left|y_{q}-1\right|\right) / 2\right)\right|_{y_{q}= \pm 1}$ do not exist. Hence, the obtained results in many references, such as [32, 33, 46, 49], cannot be applied in these functions. This leads to limitations for their results in applications whereas our work has just filled the gap. Moreover, from Assumption 1, it is easy to obtain that $\kappa_{q}^{R R}=\kappa_{q}^{I I}=\pi_{q}^{R R}=\pi_{q}^{I I}=1, \kappa_{q}^{R I}=\kappa_{q}^{I R}=\pi_{q}^{R I}=$ $\pi_{q}^{I R}=0$ for $q=1,2$. 

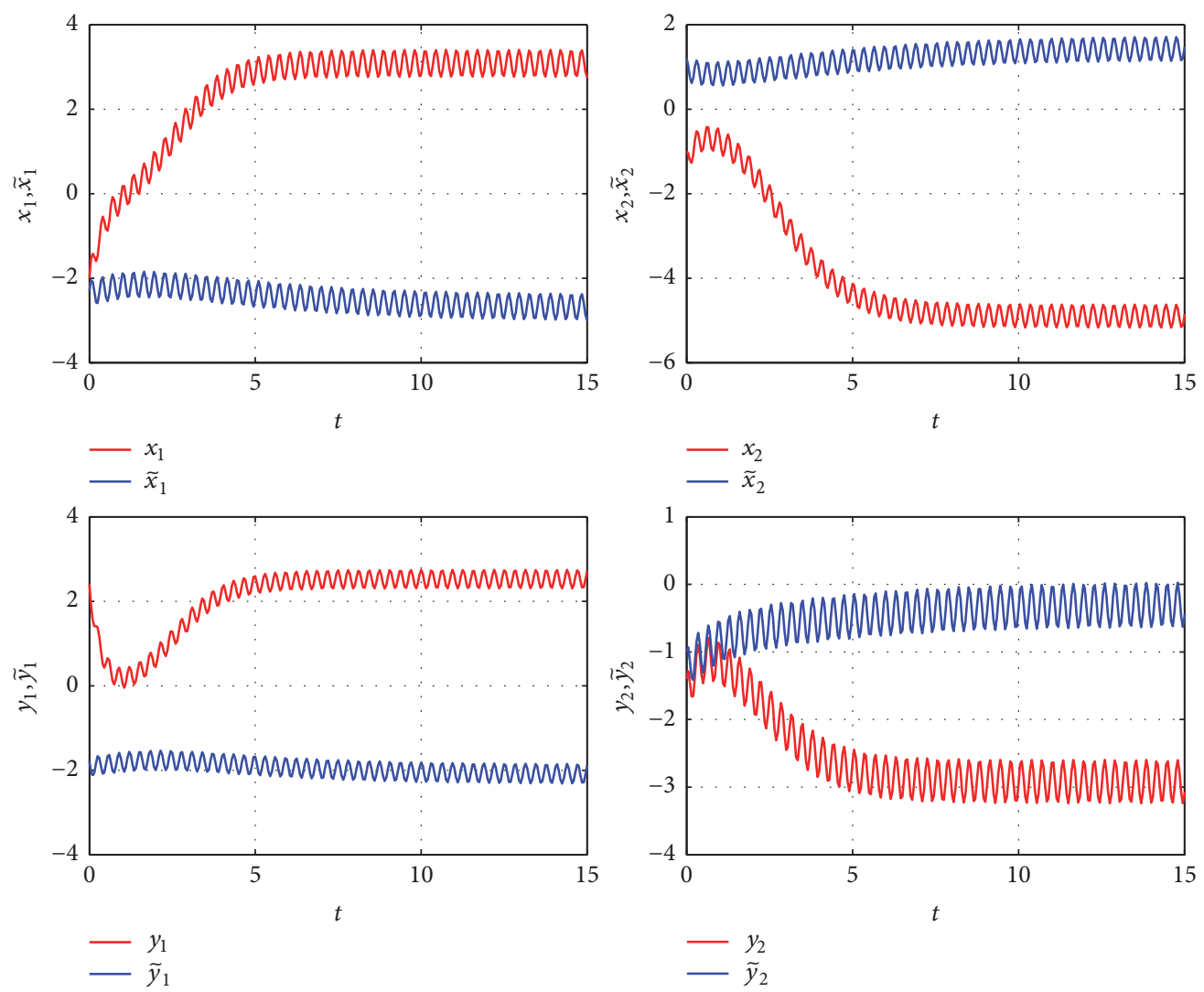

FIgURE 2: Time responses of real and imaginary parts of the variables $z_{1}, z_{2}, \widetilde{z}_{1}$, and $\widetilde{z}_{2}$ without controller for example 1.

The response system is given by

$$
\begin{aligned}
\dot{\vec{z}}_{p}(t)= & -d_{p} \widetilde{z}_{p}(t)+\sum_{q=1}^{2} a_{p q} h_{q}\left(\widetilde{z}_{q}(t)\right) \\
& +\sum_{q=1}^{2} b_{p q} m_{q}\left(\widetilde{z}_{q}\left(t-\tau_{q}\right)\right)+J_{p}(t)+u_{p}(t) .
\end{aligned}
$$

Under the initial conditions $z_{1}(\theta)=0.8-0.9 i, z_{2}(\theta)=$ $1.1-i, \widetilde{z}_{1}(\theta)=-1+i$, and $\widetilde{z}_{2}(\theta)=-0.6+1.1 i$ for $\theta \in[-0.2,0]$, Figures 5 and 6 depict the trajectories of systems (31) and (33) without controller, which shows that the drive system (31) does not synchronize with the response system (33).

By choosing the parameters of the controller (15) as $\alpha_{11}=$ 4.2, $\alpha_{12}=6.5, \alpha_{21}=4.4, \alpha_{22}=5.9, \beta_{11}=\beta_{12}=\beta_{21}=\beta_{22}=1$, $\gamma_{11}=2.5, \gamma_{12}=3.1, \gamma_{21}=2, \gamma_{22}=3.6, \mu_{1}=0.5, \mu_{2}=$ 0.6 , it is easy to check that the conditions of Theorem 10 are satisfied. Thus, under the corresponding controller, the drive system (31) can be synchronized with the response system (33) in finite time. Moreover, according to Theorem 10 and Remark 7, we get $T_{2} \leq \max \{10.95,11.19\}=11.19$ seconds by simple calculation. The synchronization curves between the drive system (31) and the response system (33) are shown in Figure 7. The time responses of the synchronization errors between them are depicted in Figure 8. From Figures 7 and 8 , it can be seen that system (31) can synchronize with system (33) in finite time via the corresponding controller under the given initial values. This example also further illustrates the effectiveness and superiority of our proposed result.

\section{Conclusion}

In this paper, the finite-time synchronization problem of complex-valued recurrent neural networks with time delays has been studied. Based on the more general assumptions for activation functions, a nonlinear controller with independent parameters, and a new inequality proposed and proved by us in [52], some sufficient conditions have been established and the settling time for synchronization has been estimated. The obtained results have been shown to be effective and superior by two examples. Moreover, it is well known that the systems with stochastic terms are more extensive in practical applications [56-60], such as the noises, and we will discuss the addressed models with noises deeply in the future.

\section{Data Availability}

No data was used in this study.

\section{Conflicts of Interest}

The authors declare that there are no conflicts of interest regarding the publication of this paper. 

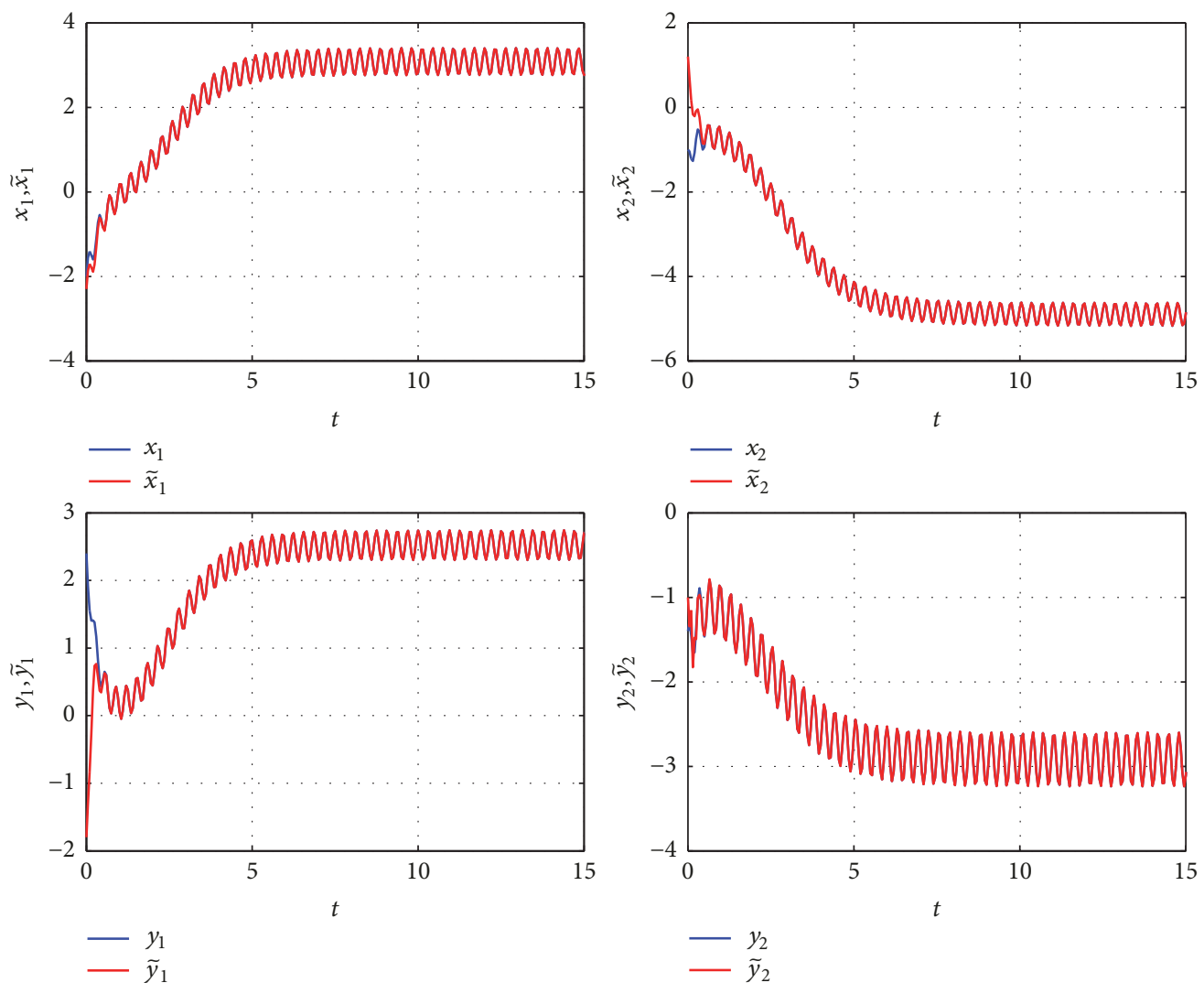

$y_{2}$
$-\tilde{y}_{2}$

FIGURE 3: Synchronization curves of real and imaginary parts of $z_{1}, z_{2}, \widetilde{z}_{1}$, and $\widetilde{z}_{2}$ under controller for example 1.
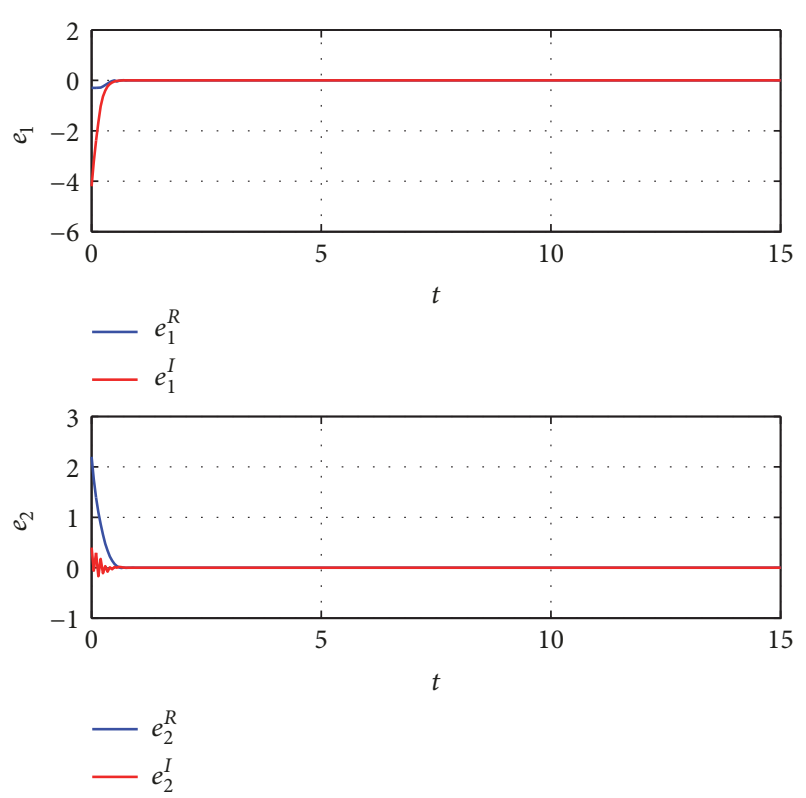

FIGURE 4: Time responses of synchronization errors $e_{1}$ and $e_{2}$ under controller for example 1.

\section{Acknowledgments}

This work was supported in part by the National Natural Science Foundation of China (61503222, 61673227, 61573008,
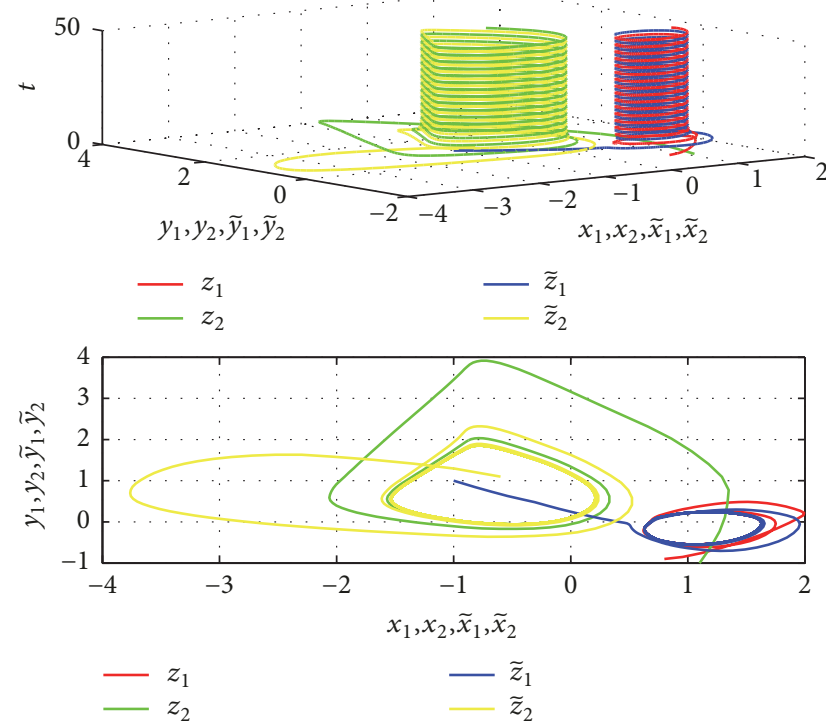

FIGURE 5: Time responses of the variables $z_{1}, z_{2}, \widetilde{z}_{1}$, and $\widetilde{z}_{2}$ in $3 \mathrm{D}$ and $2 \mathrm{D}$ spaces without controller for example 2.

61773245), in part by NSERC, Canada, in part by the Research Fund for the Taishan Scholar Project of Shandong Province of China, in part by the Project funded by Chinese Postdoctoral Science Foundation (2016M602166), and in part by the 

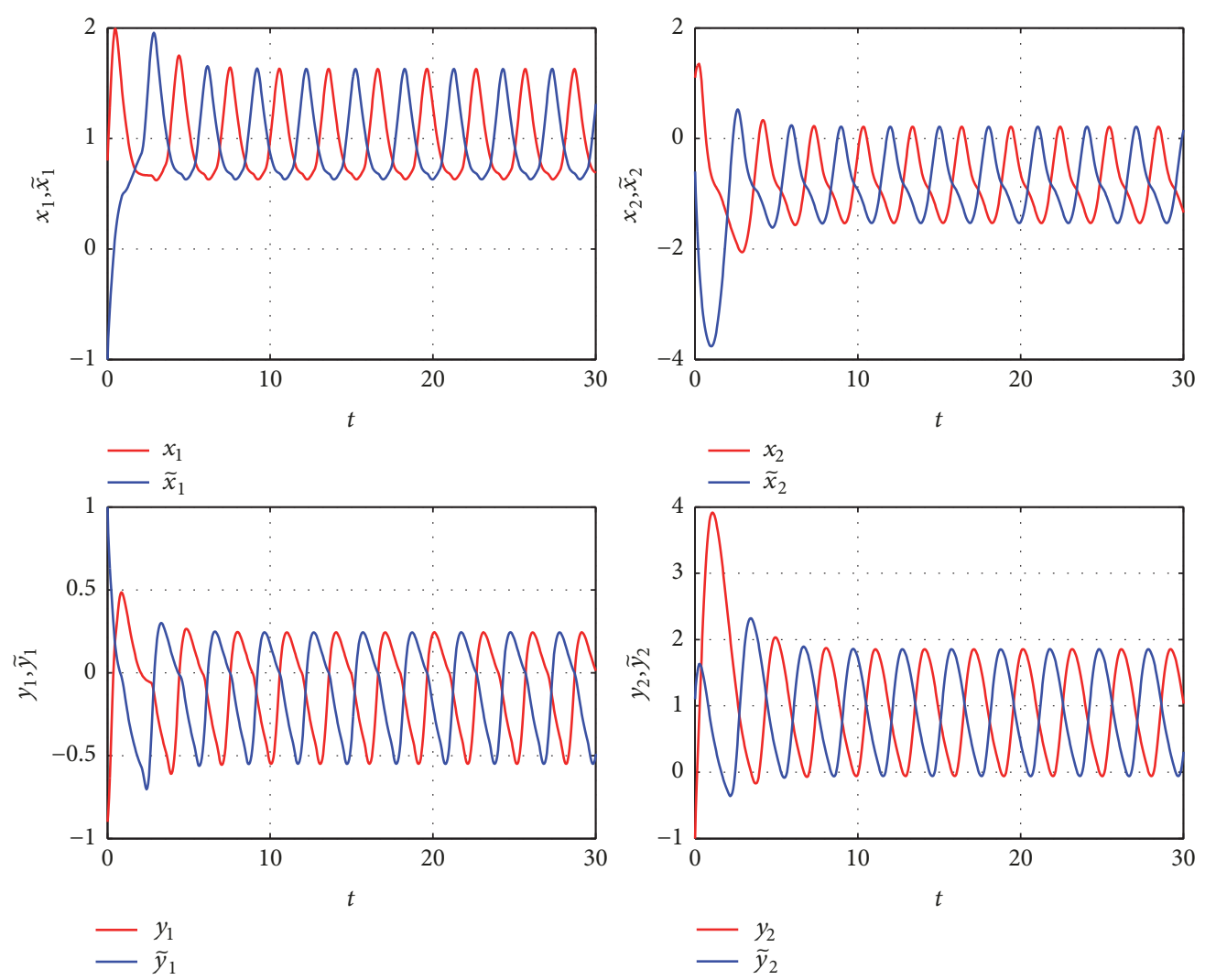

FIGURE 6: Time responses of real and imaginary parts of the variables $z_{1}, z_{2}, \widetilde{z}_{1}$, and $\widetilde{z}_{2}$ without controller for example 2 .
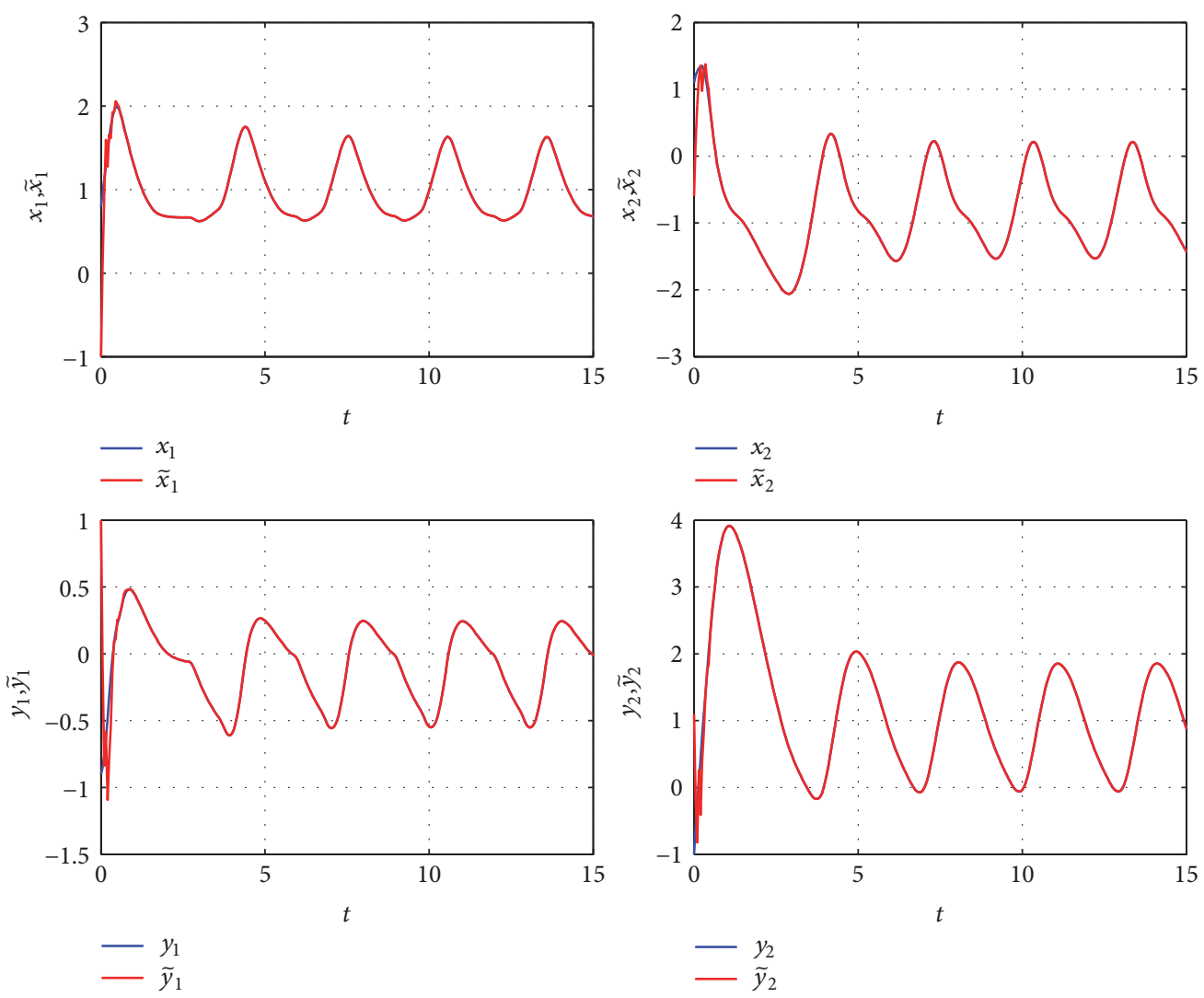

$y_{2}$
$-\tilde{y}_{2}$

FIGURE 7: Synchronization curves of real and imaginary parts of $z_{1}, z_{2}, \widetilde{z}_{1}$, and $\widetilde{z}_{2}$ under controller for example 2 . 

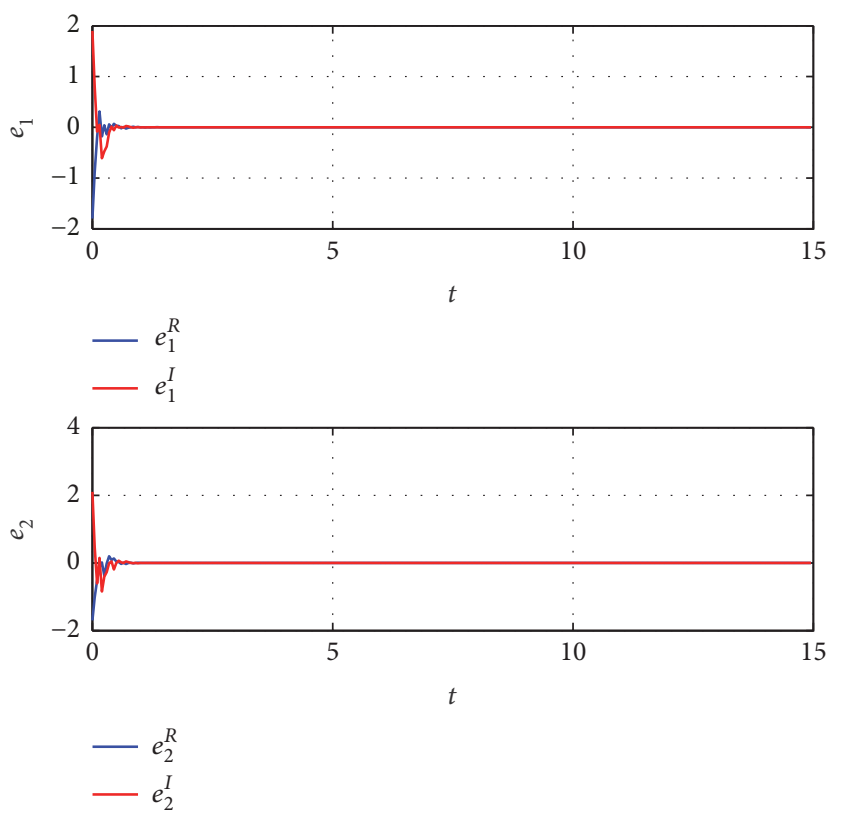

FIGURE 8: Time responses of synchronization errors $e_{1}$ and $e_{2}$ under controller for example 2.

Fund for Postdoctoral Applied Research Projects of Qingdao (2016116).

\section{References}

[1] M. Forti and A. Tesi, "New conditions for global stability of neural networks with application to linear and quadratic programming problems," IEEE Transactions on Circuits and Systems I: Fundamental Theory and Applications, vol. 42, no. 7, pp. 354-366, 1995.

[2] S. Jiao, H. Shen, Y. Wei, X. Huang, and Z. Wang, "Further results on dissipativity and stability analysis of Markov jump generalized neural networks with time-varying interval delays," Applied Mathematics and Computation, vol. 336, pp. 338-350, 2018.

[3] Y. Fan, X. Huang, Z. Wang, J. Xia, and Y. Li, "Global MittagLeffler synchronization of delayed fractional-order memristive neural networks," Advances in Difference Equations, vol. 338, 2018.

[4] M. Xing, H. Shen, and Z. Wang, " $H_{\infty}$ synchronization of semiMarkovian jump neural networks with randomly occurring time-varying delays," Complexity, vol. 2018, Article ID 8094292, 16 pages, 2018.

[5] W. Lv and F. Wang, "Adaptive tracking control for a class of uncertain nonlinear systems with infinite number of actuator failures using neural networks," Advances in Difference Equations, vol. 374, 2017.

[6] H. Wang, X. Liu, and K. Liu, "Robust adaptive neural tracking control for a class of stochastic nonlinear interconnected systems," IEEE Transactions on Neural Networks and Learning Systems, vol. 27, no. 3, pp. 510-523, 2016.

[7] N. Ozcan, M. S. Ali, J. Yogambigai, Q. Zhu, and S. Arik, "Robust synchronization of uncertain Markovian jump complex dynamical networks with time-varying delays and reaction-diffusion terms via sampled-data control," Journal of The Franklin Institute, vol. 355, no. 3, pp. 1192-1216, 2018.

[8] M. Syed Ali, Q. Zhu, S. Pavithra, and N. Gunasekaran, "A study on $\langle(\mathrm{Q}, \mathrm{S}, \mathrm{R})-\gamma\rangle$-dissipative synchronisation of coupled reaction-diffusion neural networks with time-varying delays," International Journal of Systems Science, vol. 49, no. 4, pp. 755765, 2018.

[9] S. P. Bhat and D. S. Bernstein, "Finite-time stability of continuous autonomous systems," SIAM Journal on Control and Optimization, vol. 38, no. 3, pp. 751-766, 2000.

[10] E. Moulay, M. Dambrine, N. Yeganefar, and W. Perruquetti, "Finite-time stability and stabilization of time-delay systems," Systems \& Control Letters, vol. 57, no. 7, pp. 561-566, 2008.

[11] R. Yang and Y. Wang, "Finite-time stability and stabilization of a class of nonlinear time-delay systems," SIAM Journal on Control and Optimization, vol. 50, no. 5, pp. 3113-3131, 2012.

[12] M. Ali, J. Yogambigai, and O. M. Kwon, "Finite-time robust passive control for a class of switched reaction-diffusion stochastic complex dynamical networks with coupling delays and impulsive control," International Journal of Systems Science, vol. 49, no. 4, pp. 718-735, 2018.

[13] J. Zhang, J. Xia, W. Sun, G. Zhuang, and Z. Wang, "Finite-time tracking control for stochastic nonlinear systems with full state constraints," Applied Mathematics and Computation, vol. 338, pp. 207-220, 2018.

[14] Z. Yan, G. Zhang, J. Wang, and W. Zhang, "State and output feedback finite-time guaranteed cost control of linear It stochastic systems," Journal of Systems Science and Complexity, vol. 28, no. 4, pp. 813-829, 2015.

[15] J. Sun, Y. Wang, Y. Wang, and Y. Shen, "Finite-time synchronization between two complex-variable chaotic systems with unknown parameters via nonsingular terminal sliding mode control," Nonlinear Dynamics, vol. 85, no. 2, pp. 1105-1117, 2016.

[16] J. Sun, Y. Wu, G. Cui, and Y. Wang, "Finite-time real combination synchronization of three complex-variable chaotic systems with unknown parameters via sliding mode control," Nonlinear Dynamics, vol. 88, no. 3, pp. 1677-1690, 2017.

[17] F. Xiao, L. Wang, J. Chen, and Y. Gao, "Finite-time formation control for multi-agent systems," Automatica, vol. 45, no. 11, pp. 2605-2611, 2009.

[18] L. Wang and F. Xiao, "Finite-time consensus problems for networks of dynamic agents," IEEE Transactions on Automatic Control, vol. 55, no. 4, pp. 950-955, 2010.

[19] Z. Zhang, X. Liu, Y. Liu, C. Lin, and B. Chen, "Fixed-time almost disturbance decoupling of nonlinear time-varying systems with multiple disturbances and dead-zone input," Information Sciences, vol. 450, pp. 267-283, 2018.

[20] Y. Jing, Y. Liu, and S. Zhou, "Prescribed performance finitetime tracking control for uncertain nonlinear systems," Journal of Systems Science and Complexity, vol. 31, pp. 1-15, 2018.

[21] Hu Gao I, Jianwe Xia I, Guangming Zhuang, Zhen Wang, and Qun Sun, "Nonfragile finite-time extended dissipative control for a class of uncertain switched neutral systems," Complexity, vol. 2017, Article ID 6581308, 22 pages, 2017.

[22] X. Liu, N. Jiang, J. Cao, S. Wang, and Z. Wang, "Finitetime stochastic stabilization for BAM neural networks with uncertainties," Journal of The Franklin Institute, vol. 350, no. 8, pp. 2109-2123, 2013.

[23] L. Wang, Y. Shen, and G. Zhang, "Finite-time stabilization and adaptive control of memristor-based delayed neural networks," IEEE Transactions on Neural Networks and Learning Systems, vol. 28, no. 11, pp. 2648-2659, 2017. 
[24] L. M. Wang and Y. Shen, "Finite-time stabilizability and instabilizability of delayed memristive neural networks with nonlinear discontinuous controller," IEEE Transactions on Neural Networks and Learning Systems, vol. 26, no. 11, pp. 2914-2924, 2015.

[25] M. S. Ali and S. Saravanan, "Robust finite-time Ho control for a class of uncertain switched neural networks of neutral-type with distributed time varying delays," Neurocomputing, vol. 177, pp. 454-468, 2016.

[26] T. Wang, S. Zhao, W. Zhou, and W. Yu, "Finite-time state estimation for delayed Hopfield neural networks with Markovian jump," Neurocomputing, vol. 156, pp. 193-198, 2015.

[27] A. Abdurahman, H. Jiang, and Z. Teng, "Finite-time synchronization for fuzzy cellular neural networks with time-varying delays," Fuzzy Sets and Systems, vol. 297, pp. 96-111, 2016.

[28] F. Yang, J. Mei, and Z. Wu, "Finite-time synchronisation of neural networks with discrete and distributed delays via periodically intermittent memory feedback control," IET Control Theory \& Applications, vol. 10, no. 14, pp. 1630-1640, 2016.

[29] X. Liu, J. Cao, W. Yu, and Q. Song, "Nonsmooth finite-time synchronization of switched coupled neural networks," IEEE Transactions on Cybernetics, vol. 46, no. 10, pp. 2360-2371, 2016.

[30] A. Hirose, Complex-Valued Neural Networks: Theories and Applications, World Scientific, Singapore, 2013.

[31] Z. Zhang, R. Guo, X. Liu, and C. Lin, "Lagrange exponential stability of complex-valued BAM neural networks with timevarying delays," IEEE Transactions on Systems, Man, and Cybernetics: Systems, vol. 99, pp. 1-14, 2018.

[32] J. Hu and J. Wang, "Global stability of complex-valued recurrent neural networks with time-delays," IEEE Transactions on Neural Networks and Learning Systems, vol. 23, no. 6, pp. 853-865, 2012.

[33] X. Liu and T. Chen, "Global exponential stability for complexvalued recurrent neural networks with asynchronous time delays," IEEE Transactions on Neural Networks and Learning Systems, vol. 27, no. 3, pp. 593-606, 2016.

[34] M. E. Valle, "Complex-valued recurrent correlation neural networks," IEEE Transactions on Neural Networks and Learning Systems, vol. 25, no. 9, pp. 1600-1612, 2014.

[35] Z. Zhang, C. Lin, and B. Chen, "Global stability criterion for delayed complex-valued recurrent neural networks," IEEE Transactions on Neural Networks and Learning Systems, vol. 25, no. 9, pp. 1704-1708, 2014.

[36] R. Guo, Z. Zhang, X. Liu, and C. Lin, "Existence, uniqueness, and exponential stability analysis for complex-valued memristor-based BAM neural networks with time delays," Applied Mathematics and Computation, vol. 311, pp. 100-117, 2017.

[37] D. Xu and M. Tan, "Delay-independent stability criteria for complex-valued BAM neutral-type neural networks with time delays," Nonlinear Dynamics, vol. 89, no. 2, pp. 819-832, 2017.

[38] Z. Zhang, X. Liu, J. Chen, R. Guo, and S. Zhou, "Further stability analysis for delayed complex-valued recurrent neural networks," Neurocomputing, vol. 251, pp. 81-89, 2017.

[39] T. Dong, X. Liao, and A. Wang, "Stability and Hopf bifurcation of a complex-valued neural network with two time delays," Nonlinear Dynamics, vol. 82, no. 1-2, pp. 173-184, 2015.

[40] L. Li, Z. Wang, Y. Li, H. Shen, and J. Lu, "Hopf bifurcation analysis of a complex-valued neural network model with discrete and distributed delays," Applied Mathematics and Computation, vol. 330, pp. 152-169, 2018.
[41] Y. Liu, D. Zhang, and J. Lu, "Global exponential stability for quaternion-valued recurrent neural networks with timevarying delays," Nonlinear Dynamics, vol. 87, no. 1, pp. 553-565, 2017.

[42] Z. Wang, X. Wang, Y. Li, and X. Huang, "Stability and Hopf bifurcation of fractional-order complex-valued single neuron model with time delay," International Journal of Bifurcation and Chaos, vol. 27, no. 13, Article ID 1750209, 2017.

[43] R. Guo, Z. Zhang, and M. Gao, "State estimation for complexvalued memristive neural networks with time-varying delays," Advances in Difference Equations, vol. 118, 2018.

[44] X. Li, R. Rakkiyappan, and G. Velmurugan, "Dissipativity analysis of memristor-based complex-valued neural networks with time-varying delays," Information Sciences, vol. 294, pp. 645-665, 2015.

[45] G. Velmurugan, R. Rakkiyappan, and S. Lakshmanan, "Passivity analysis of memristor-based complex-valued neural networks with time-varying delays," Neural Processing Letters, vol. 42, no. 3, pp. 517-540, 2015.

[46] H. Wang, S. Duan, T. Huang, L. Wang, and C. Li, "Exponential stability of complex-valued memristive recurrent neural networks," IEEE Transactions on Neural Networks and Learning Systems, vol. 28, no. 3, pp. 766-771, 2017.

[47] D. Liu, S. Zhu, and W. Chang, "Global exponential stability of stochastic memristor-based complex-valued neural networks with time delays," Nonlinear Dynamics, vol. 90, no. 2, pp. 915934, 2017.

[48] R. Guo, Z. Zhang, X. Liu, C. Lin, H. Wang, and J. Chen, "Exponential input-to-state stability for complex-valued memristorbased BAM neural networks with multiple time-varying delays," Neurocomputing, vol. 275, pp. 2041-2054, 2018.

[49] R. Rakkiyappan, G. Velmurugan, and J. Cao, "Finite-time stability analysis of fractional-order complex-valued memristorbased neural networks with time delays," Nonlinear Dynamics, vol. 78, no. 4, pp. 2823-2836, 2014.

[50] Z. Zhang, X. Liu, R. Guo, and C. Lin, "Finite-time stability for delayed complex-valued bam neural networks," Neural Processing Letters, vol. 48, no. 1, pp. 179-193, 2018.

[51] C. Zhou, W. Zhang, X. Yang, C. Xu, and J. Feng, "Finitetime synchronization of complex-valued neural networks with mixed delays and uncertain perturbations," Neural Processing Letters, vol. 46, no. 1, pp. 271-291, 2017.

[52] Z. Zhang, X. Liu, D. Zhou, C. Lin, J. Chen, and H. Wang, "Finite-time stabilizability and instabilizability for complexvalued memristive neural networks with time delays," IEEE Transactions on Systems, Man, and Cybernetics: Systems, vol. 99, pp. 1-12, 2017.

[53] Y. Wang, Z. Pan, Y. Li, and W. Zhang, " $H_{\infty}$ control for nonlinear stochastic markov systems with time-delay and multiplicative noise," Journal of Systems Science and Complexity, vol. 30, no. 6, pp. 1-23, 2017.

[54] J. Chen, T. Zhang, Z. Zhang, C. Lin, and B. Chen, "Stability and output feedback control for singular Markovian jump delayed systems," Mathematical Control and Related Fields, vol. 8, no. 2, pp. 475-490, 2018.

[55] M. Gao, L. Sheng, and W. Zhang, " $H_{2} / H_{\infty}$ control of nonlinear systems with time-delay and state-dependent noise," Applied Mathematics and Computation, vol. 266, pp. 429-440, 2015.

[56] J. Wang, K. Liang, X. Huang, Z. Wang, and H. Shen, "Dissipative fault-tolerant control for nonlinear singular perturbed systems with Markov jumping parameters based on slow state feedback," 
Applied Mathematics and Computation, vol. 328, pp. 247-262, 2018.

[57] W. Zhang, L. Ma, and T. Zhang, "Discrete-time mean-field stochastic $\mathrm{H}_{2} / \mathrm{H}_{\mathrm{\infty}}$ control," Journal of Systems Science and Complexity, vol. 30, no. 4, pp. 765-781, 2017.

[58] S. Zhou, X. Liu, B. Chen, and H. Liu, "Stability analysis for a class of discrete-time nonhomogeneous markov jump systems with multiplicative noises," Complexity, vol. 2018, Article ID 1586846, 9 pages, 2018.

[59] K. Liang, M. Dai, H. Shen, J. Wang, Z. Wang, and B. Chen, " $L_{2}{ }^{-}$ $L_{\infty}$ synchronization for singularly perturbed complex networks with semi-Markov jump topology," Applied Mathematics and Computation, vol. 321, pp. 450-462, 2018.

[60] Y. Zhao and W. Zhang, "Observer-based controller design for singular stochastic Markov jump systems with state dependent noise," Journal of Systems Science \& Complexity, vol. 29, no. 4, pp. 946-958, 2016. 


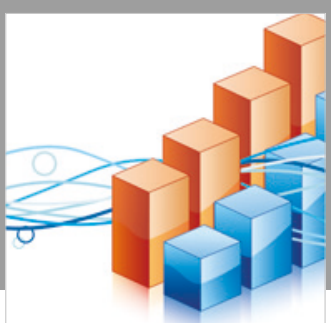

Advances in

Operations Research

\section{-n-m}
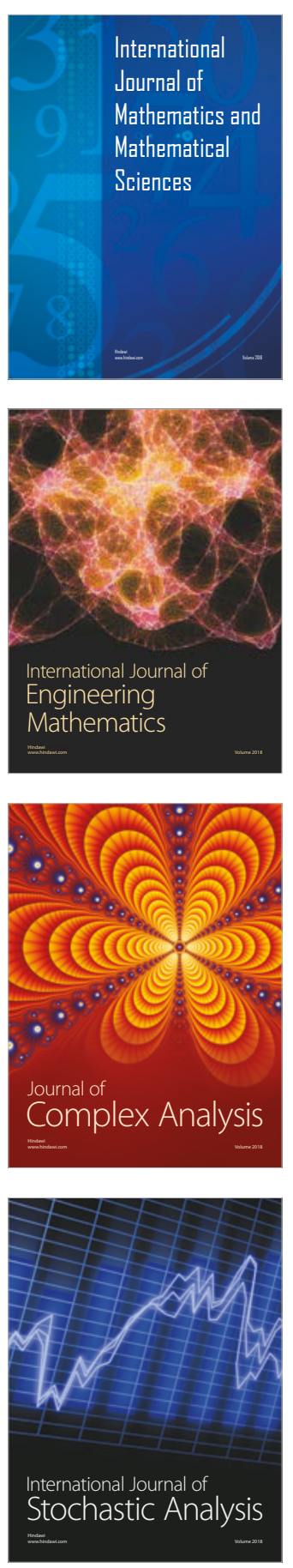
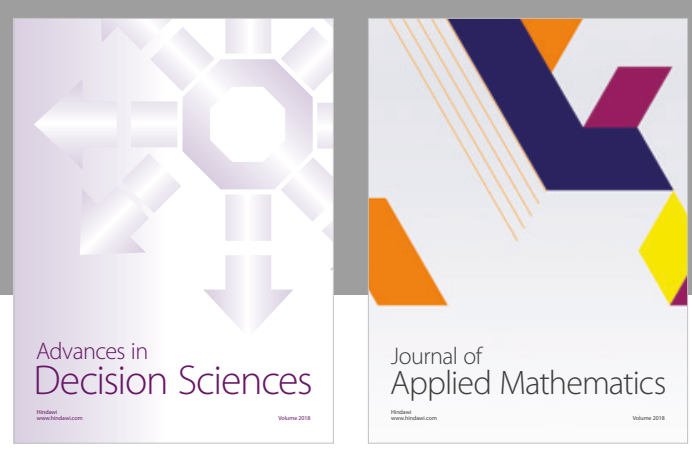

Journal of

Applied Mathematics
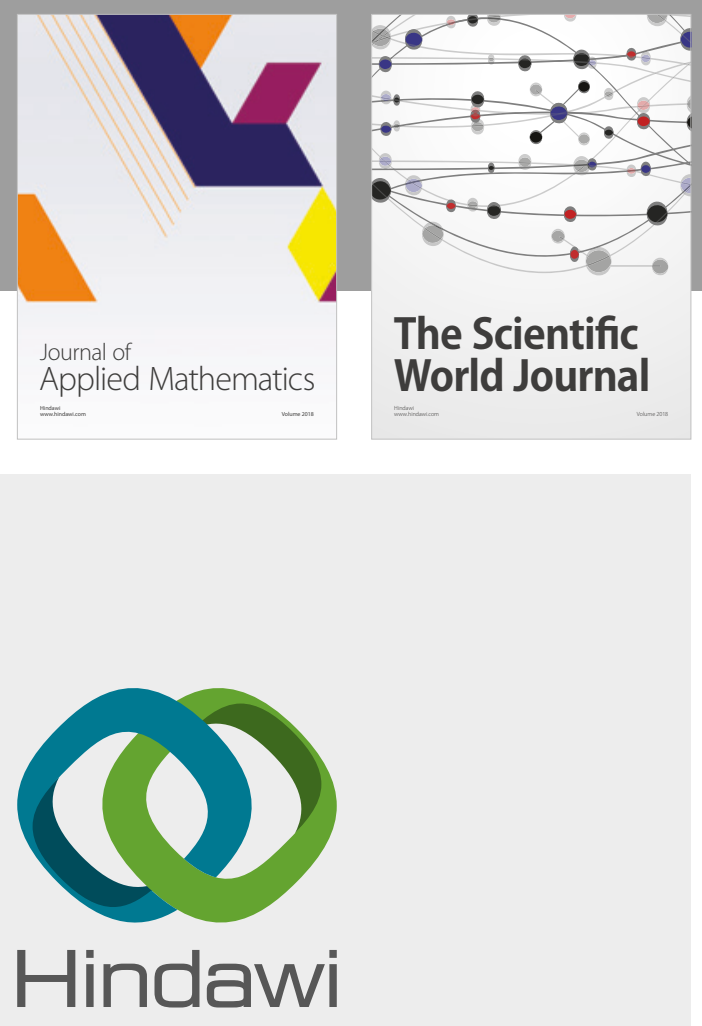

Submit your manuscripts at

www.hindawi.com

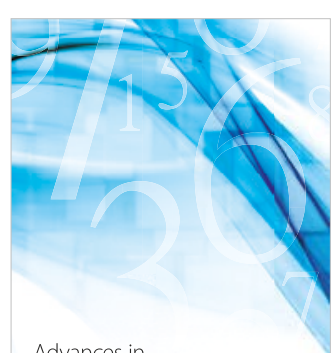

Advances in
Numerical Analysis
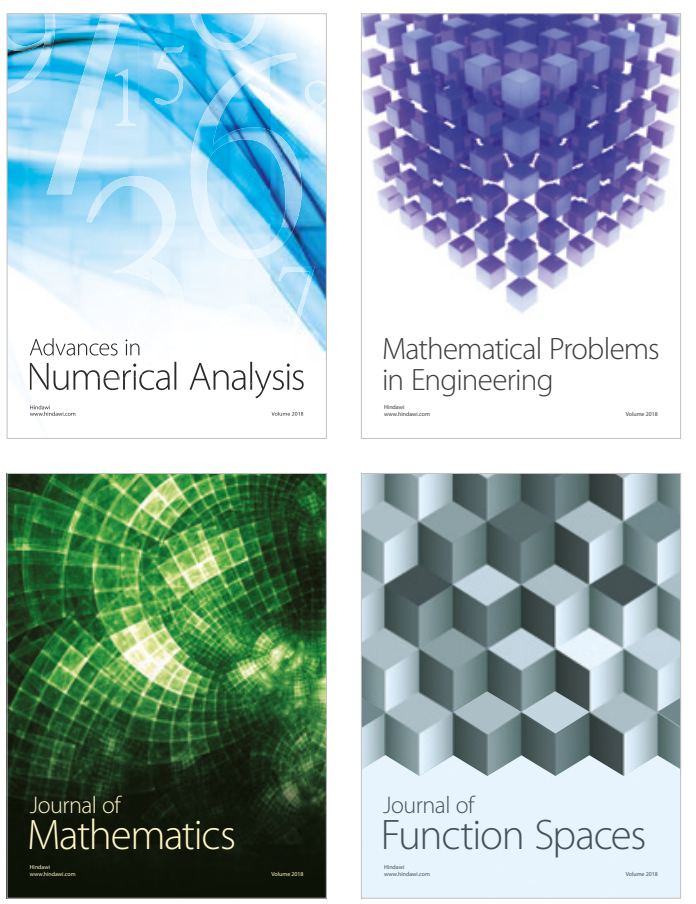

Mathematical Problems in Engineering

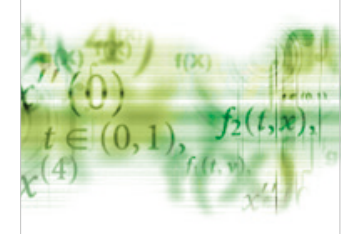

International Journal of

Differential Equations

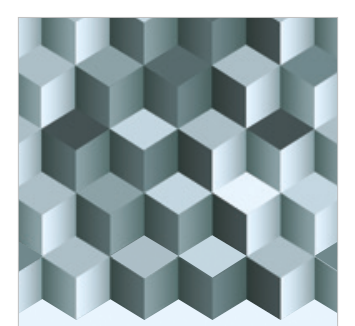

Journal of

Function Spaces
The Scientific

World Journal

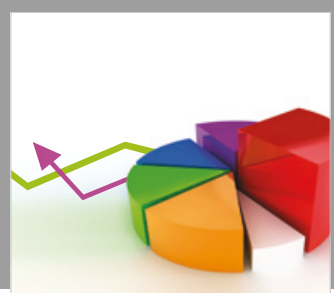

Journal of

Probability and Statistics
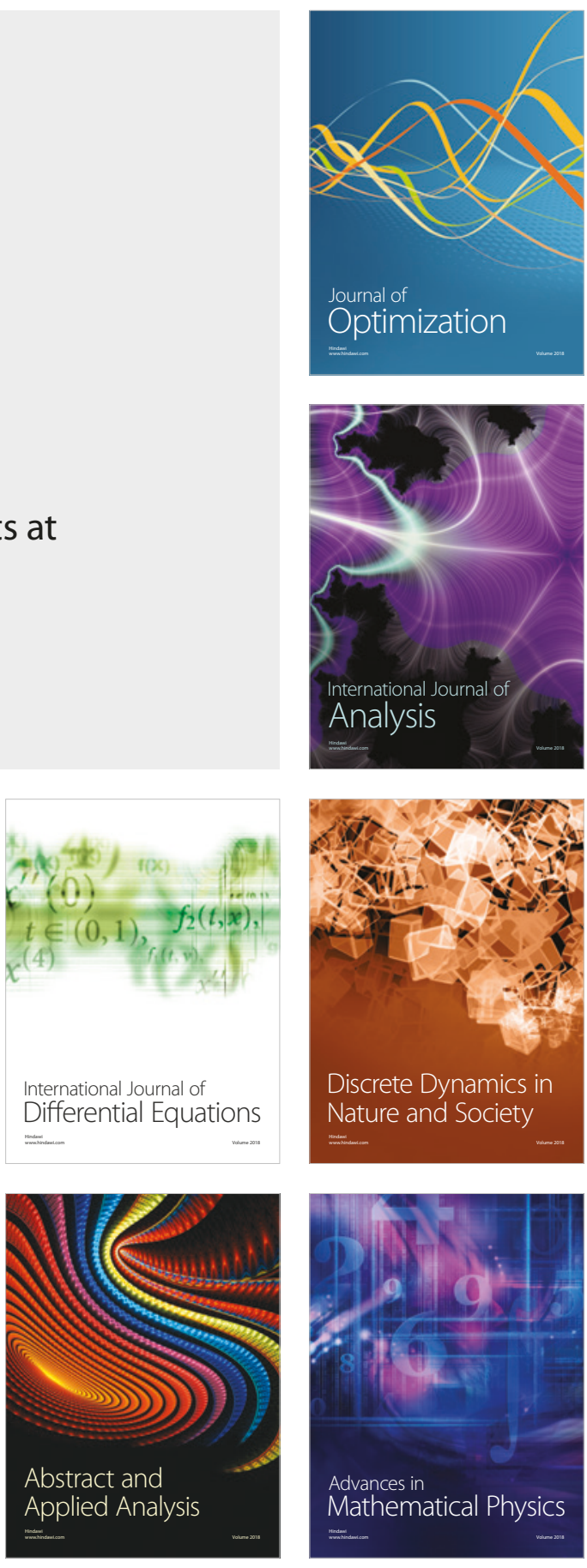